Published in final edited form as:

J Cancer Surviv. 2016 October ; 10(5): 814-831. doi:10.1007/s11764-016-0527-6.

\title{
Psychosocial outcomes and interventions among cancer survivors diagnosed during adolescence and young adulthood (AYA): a systematic review
}

\author{
Marie Barnett ${ }^{1}$, Glynnis McDonnell ${ }^{2,3}$, Antonio DeRosa ${ }^{2}$, Tammy Schuler ${ }^{2,4}$, Errol Philip ${ }^{5}$, \\ Lisa Peterson ${ }^{2}$, Kaitlin Touza ${ }^{6}$, Sabrina Jhanwar ${ }^{2}$, Thomas M. Atkinson ${ }^{2}$, and Jennifer S. \\ Ford $^{2}$ \\ ${ }^{1}$ Columbia University Medical Center, New York, NY, USA \\ ${ }^{2}$ Department of Psychiatry \& Behavioral Sciences, Memorial Sloan Kettering Cancer Center, 641 \\ Lexington Ave., 7th Floor, New York, NY 10022, USA \\ ${ }^{3}$ St. John's University Department of Psychology, Queens, NY, USA \\ ${ }^{4}$ Association for Behavioral and Cognitive Therapies, New York, NY, USA \\ ${ }^{5}$ The Notre Dame Laboratory for Psycho-oncology Research, Notre Dame, IN, USA \\ ${ }^{6}$ Indiana University-Purdue University Indianapolis, Indianapolis, IN, USA
}

\section{Abstract}

Purpose-A cancer diagnosis during adolescence or young adulthood (AYA; defined as ages 1539) generates unique medical and psychosocial needs as developmental milestones are simultaneously impacted. Past research highlights that AYAs' experiences and psychosocial outcomes are different, and more research and attention is needed. We aimed to identify and synthesize literature regarding psychosocial outcomes, unique needs, and existing psychosocial interventions pertaining to individuals diagnosed with cancer exclusively during AYA, and to highlight areas for future research.

Methods-A systematic literature search was conducted using MEDLINE (via PubMed), EMBASE, Cochrane, Web of Science, and PsycINFO (via OVID). Grey literature was searched using key term variations and combinations. Overall, 15,301 records were assessed by two independent reviewers, with 38 studies meeting inclusion criteria.

Jennifer S. Ford, fordj@mskcc.org.

Marie Barnett, marie.barnett@gmail.com

Glynnis McDonnell, glynnis.mcdonnell14@ @stjohns.edu

Antonio DeRosa, derosaa1@mskcc.org

Tammy Schuler, tschuler@abct.org

Errol Philip, ejphilip@gmail.com

Lisa Peterson, petersol@mskcc.org

Kaitlin Touza, ktouza@iupui.edu

Sabrina Jhanwar, jhanwas1@mskcc.org

Thomas M. Atkinson, atkinsot@mskcc.org

Compliance with ethical standards

Conflict of interest The authors declare that they have no conflicts of interest. 
Results-Data synthesis of the 38 articles was organized by four main themes based on quality of life and survivorship: physical well-being ( 7 studies), psychological well-being (8 studies), social well-being ( 9 studies), and survivorship care (14 studies). The paucity of studies for such broad inclusion criteria highlights that this population is often combined or subsumed under other age groups, missing needs unique to these AYAs.

Conclusions-AYA cancer survivors' experiences are nuanced, with interacting variables contributing to post-treatment outcomes. AYAs require age-appropriate and flexible care, informational needs and treatment-related education that foster autonomy for long-term survivorship, as well as improved follow-up care and psychological outcomes.

Implications for Cancer Survivors-By incorporating these findings into practice, the informational and unmet needs of AYAs can be addressed effectively. Education and programming is lacking specific and general subject matter specific to AYAs, incorporating ranging needs at different treatment stages.

\section{Keywords}

Adolescent; Cancer survivorship; Literature review; Neoplasms; Young adult

\section{Introduction}

A diagnosis of cancer during adolescence and young adulthood (i.e., "AYAs") can generate medical and psychosocial needs and consequences unique to this age group [1,2]. While the majority of past research has focused on survivors of childhood cancers, more people between the ages of 15-39 years of age are diagnosed with cancer than those in the first 15 years of life, and these rates are increasing [3].

Survivors of cancer diagnosed in adolescence or young adulthood will likely engender different experiences than those diagnosed as children or older adults. For example, a key feature within the adolescent population (i.e., individuals ages 15-18) is the intersection of the cancer experience with a period of rapid biological and psychosocial development. Specific developmental constructs which may be challenged for this group include autonomy and independence, peer pressure, social and sexual maturation, fertility, and finances [4]. Moreover, adverse and late effects of treatment can physically impact survivors, as well as cause short- and long-term effects on their self-image and well-being [5, 6]. Furthermore, young adults (i.e., individuals ages 19-39) also present with unique challenges regarding the interaction between the cancer experience and psychosocial development. Cancer diagnosis and treatment may present unexpected challenges and choices in the context of a new marriage/partnership, educational or occupational pursuits, finances, or child rearing. Consequently, whereas prior reviews of the literature have highlighted the need for further exploration and clarification of outcomes within the AYA population, given that this specific age group has been typically assessed in combination with younger and/or older ages [7], this systematic review aimed to identify relevant psychosocial outcomes, specific needs, and existing psychosocial interventions pertaining to those diagnosed with cancer exclusively during AYA. 


\section{Method}

\section{Literature search}

A systematic search for articles was conducted using MEDLINE (via PubMed), Embase, Cochrane, Web of Science, and PsycINFO (via OVID). There were no date, language, or publication type restrictions; each database was search in its entirety, through March 2014. With the exception of Web of Science, which does not employ a controlled vocabulary, both controlled vocabulary and text words were used in the development of the database search strategy.

The search strategy had three components, with all concepts linked together via the AND operator: (1) cancer survivors, survivorship; (2) psychosocial, behavior therapy, alternative therapy, interventions; and (3) outcomes, unique needs, adjustment. After combining the concepts in all five databases, the following keyword and controlled vocabulary terms (where applicable) were added to the search using the AND operator in an effort to be as comprehensive as possible: adolescent, young adult, AYA (Table 2 Appendix). Using the filter option to limit results by age group was more limiting than adding a separate concept with these terms to the overall search. The decision was made to retrieve a greater number of results rather than possibly eliminating relevant records.

Grey literature sources were also searched using variations and combinations of the following terms: cancer survivor*, psychosocial, intervention*, treatment*, adolescent*, young adult*, AYA, outcome*, result*, needs, and adjustment. The resources searched were: National Cancer Institute's (NCI) Report of the Adolescent and Young Adult Oncology Progress Review Group (http://www.cancer.gov/cancertopics/aya/reports), National Coalition for Cancer Survivorship (http://www.canceradvocacy.org), American Psychosocial Oncology Society (APOS) conference proceedings (http://www.apos-society.org), International Psycho-Oncology Society (IPOS) conference proceedings (http://www.ipossociety.org), New York Academy of Medicine (NYAM) Grey Literature Report (http:// www.greylit.org), and Online Computer Library Center (OCLC) WorldCat (for theses/ dissertations) (http://www.worldcat.org). An advanced search (or equivalent) feature was used wherever possible (OCLC WorldCat, NYAM Grey Literature Report) to ensure that the most targeted results were retrieved. All grey literature search results were compiled in a separate document and are included in the PRISMA Flow Diagram (Fig. 1) in the number of "additional records identified" box under the "Identification" phase of the overall review process.

\section{Study inclusion criteria}

Studies were deemed eligible for inclusion if they included an original characterization of psychosocial outcomes and/or interventions in patients who are survivors of cancer initially diagnosed between ages 15-39.

\section{Study selection and characteristics}

All titles were independently reviewed for eligibility by two co-authors (from a pool of seven possible reviewers). Next, each potentially eligible article was randomly assigned to a 
pair of co-authors for full abstract screening. For the full-text review phase, the randomly assigned author teams consisted of a primary reviewer and a secondary reviewer for the purposes of verification and quality assurance. Both reviewers independently completed standardized coding forms to extract the pre-determined information from each potentially eligible article. All reviewers then met as a group and compared full-text article reviews to resolve any potential discrepancies and make final decisions regarding article inclusion. Each author independently searched references from the included full-text articles to determine whether they should be also considered for inclusion.

Study quality and risk-of-bias was assessed using a modified version of the Downs and Black Study Quality checklist [46]. All included articles were reviewed and rated for the following nine quality indicators: (1) Is the hypothesis/aim/objective of the study clearly described?; (2) Are the main outcomes to be measured clearly described in the Introduction or Methods section?; (3) Are the characteristics of the patients included in the study clearly described?; (4) Are the main findings of the study clearly described?; (5) Have actual probability values been reported from the main outcomes except where the probability value is less than 0.001?; (6) Were the subjects asked to participate in the study representative of the entire population from which they were recruited?; (7) Were those subjects who were prepared to participate representative of the entire population from which they were recruited?; (8) If any of the results of the study were based on "data dredging", was this made clear?; and (9) Were the main outcome measures used accurate (valid and reliable)? Studies were categorized as "high quality" if they satisfied at least 7 of the 9 quality indicators.

\section{Results}

Database searches initially identified 16,493 articles, with an additional 24 identified via grey literature searching. After duplicates were removed, 15,301 article titles were each reviewed by two independent reviewers for eligibility. Following title review, it was determined that 1,715 unique article abstracts would be reviewed. A total of 648 articles were retained for the full text-review. A total of $610(94 \%)$ full-text articles were excluded for the following reasons: outside age eligibility $(k=465)$, poster/presentation $(k=65)$, editorial/review paper $(k=28)$, non-empirical $(k=22)$, foreign language without translation ( $k=13)$, dissertation $(k=10)$, duplicate $(k=7)$, or unable to confirm eligibility from study authors $(k=1)$. Two studies made use of identical datasets; we retained the study that best characterized this dataset to avoid double counting [47]. Poster/presentation abstracts and dissertations, while included in the initial database and grey literature search, were excluded from this review due to lack of specificity of diagnosis age. A total of 38 articles met inclusion criteria (Fig. 1).

\section{Study characteristics}

Table 1 includes a summary of participant demographics and study characteristics for the 38 included articles. Thirty-three studies utilized a cross-sectional design, five utilized prospective methods, and two were randomized intervention or feasibility studies. Studies spanned a range of methodologies, including nine using large population or registry-based 
survey databases (e.g., SEER cancer registries, Behavioral Risk Factor Surveillance System), multi-center studies, and 17 qualitative analyses. Cancer diagnoses were mixed in 30 studies, with the remaining eight single diagnosis studies. Five studies were exclusively female, with one exclusively male. Six international studies were included (Canada [ $k=4]$, Australia $[k=1]$, Germany $[k=1])$. Results were synthesized into four sections based on a quality of life (QOL) model and survivorship care recommendations as outlined in the Institute of Medicine's (IOM) report [48]: (1) physical well-being and symptoms, (2) psychological well-being, (3) social well-being, and (4) survivorship care. While sufficient quantitative data was not included in studies to permit completion of a meta-analysis, studies often described results overlapping with these sections. As such, we report how many studies were primarily and secondarily included within each section.

\section{Physical well-being and symptoms}

Physical well-being is defined by an individual's functional/physical activities, health behaviors, and knowledge of health and/or disease [48]. Symptoms also include strength/ fatigue, sleep/rest, overall physical health, diet, fertility, and pain. These symptoms are known to impact overall well-being, and for cancer survivors can be significantly impacted by treatment side effects or late-effects [5]. Physical well-being was reflected primarily in seven $(18 \%)$ studies and two secondarily.

A large-scale health-based registry comparing AYAs with and without a cancer history found that AYA survivors have poorer health behaviors and compared with same-age controls [39]. Current smoking was significantly greater among AYA survivors (26\%) compared to controls (18\%), although no difference in binge drinking rates (14 vs. $15 \%)$. More AYA survivors reported no leisure-time physical activity in the past month (31 vs. $24 \%$ ). Another national health survey found that compared to female healthy controls, female survivors reported poorer physical health, and were more likely to meet criteria for lifetime and current medical conditions, greater health-related disabilities, and greater functional limitations [25].

Psychosexual, fertility, and body image sequelae were found among male and female AYAs [9, 17, 22, 24]. Keim-Malpass and colleagues [22] reported relevance and gender-specific effects of sexual sequelae of diagnosis/treatment (e.g., functioning, fertility, gender norms). AYAs reported significantly lower health-related quality of life compared to those diagnosed at younger ages, leukemia or sarcoma survivors, or healthy controls [49].

Several studies reported specialized structured programming needs for AYA survivors, including the need for supportive care, information concerning health promotion, the cancer treatment they had undergone, nutrition, dealing with insurance, and other survivorship concerns (sexuality, fertility) [10, 36, 41]. This information was utilized to develop and test an information-based workshop series addressing the unmet needs among young breast cancer survivors [10]. After 6 months, intervention did not increase participants' cancerrelated knowledge or self-reported physical activity [10]. Rabin [37], Valle [41] and respective colleagues reported on the development and pilot testing of internet-based interventions targeting physical activity among young adult survivors. Both found that interventions utilizing social media and online components were both feasible and 
acceptable to this group of survivors. Compared to childhood survivors' preferences, AYAs do not prefer camp-based intervention delivery to improve diet and exercise behaviors [8].

Access to and use of alternative or integrative services has been established as effective with cancer patients and survivors [50, 51]; however, little research has focused on these benefits among this age-specific cohort. Park and colleagues [34] found that $37 \%$ AYAs used complementary medicine approaches since their diagnosis, including yoga to promote flexibility and relaxation.

\section{Psychological well-being}

Psychological well-being was reflected primarily in eight (21\%) studies and eight secondarily. The IOM defines this QOL section as areas of psychological and mental health, including: control, anxiety, depression, enjoyment/leisure, cognition/attention, or psychopharmacological use [48]. Psychological well-being additionally includes AYA's fear of recurrence or being different, feeling different, cancer-related disclosure, distress of diagnosis, and control of treatment.

Unmet emotional and psychological needs were reported as major concerns for AYAs after post-treatment [29, 38]. Overall, AYA survivors reported $\geq 14$ days of poor mental health in the past 30 days ( 20 vs. $10 \%$ ) compared to healthy controls ( 20 vs. $10 \%$ ) [39]. Another study found survivors to be well-adjusted, with stress and social support as significant predictors of posttraumatic growth [38]. The relationship between stress (negative relationship) social support (positive relationship) and post-traumatic growth was moderated by level of physical activity; the relationship with social support and psychological growth was stronger for individuals with lower levels of physical activity [25].

Individuals diagnosed between the ages of 15 and 20 were nearly twice as likely to use antidepressant medication as individuals diagnosed before age 5, and AYA survivors reported symptoms of post-traumatic stress, anxiety, and depression, as well as fears of recurrence $[14,15,22,26]$. Female AYAs scored significantly more poorly than healthy peers on seven out of eight mental health outcomes and were more likely than peers to meet criteria for serious psychological distress [35], and in another study reported experiencing persistent struggles, such as post-traumatic stress symptoms, anxiety, pain, and fatigue [22]. In a large survey study, more than half of AYAs who needed mental health services did not receive them, and emotional problems interfering with social activities were associated with unmet service and information needs [20]. From an international perspective, almost half of survivors in a German study reported receiving psychosocial care during or immediately post-treatment; and among those reporting clinical symptoms, only $12 \%$ received psychological help and $14 \%$ psychotropic medication [15]. Additionally, $75 \%$ of AYA survivors reported the desire for a support group [20,26]. Overall, higher psychosocial stress increased the likelihood of obtaining psychosocial treatment and psychotropic medication, and AYAs diagnosed with a second malignancy were more likely to receive psychosocial treatment. Older AYAs were more likely to follow-up with oncological care on the recommended schedule. Controlling for treatment status, predictors of depression include physical or daily living needs, and health system and information needs [16]. 
Disease and treatment features also affected psychosocial outcomes and treatment-seeking [41, 52]. Cancer diagnosis is a potential moderator of being in school or employment at follow-up, with ALL and non-Hodgkin's Lymphoma diagnoses less likely to be in one of these groups compared with other diagnoses [9,34]. AYAs experiencing cancer-related education or work interruption were significantly more distress [42]. Individuals who required intensive care or stem cell transplant were more likely to receive psychosocial treatment. AYAs greater than 2 years post-treatment reported worsened psychosocial wellbeing and functioning, and decreased positive attitude towards care [11].

Coping at each phase presents new challenges: diagnosis phase (i.e., information, relational interactions, and perceptions), treatment phase (i.e., taking action, control, adaptation, situation self), and post-treatment/remission/palliative phase (i.e., normalcy) [27]. AYAs report a lack of survivorship preparation (especially from healthcare providers), late effects that pervade their entire life, a lack of post-treatment understanding from social networks, and difficulty coming to terms being "neither sick nor healthy" [19]. A qualitative analysis of young women's online narratives found four themes describing the transition into longterm survivorship: living in the middle (after treatment but before "remission"), new normal, urgency, and transition into the abyss [21]. Specifically, osteosarcoma survivors reported that cancer treatment caused them to redefine their personal and professional work into different categories (i.e., illness work, identity work, vocational work), ultimately altering their pretreatment goals [33].

Among breast cancer survivors, treatment decisions were greatly motivated and determined by survival chances; however as noted above, fertility options, services, and empowerment were either inadequate or provided too late during and post-treatment [17, 24]. Women reported persistent struggle regarding fertility concerns [22]. Both women and men who received bone marrow transplant or chemotherapy reported poorer body image, and women reported lower frequency of sexual behaviors, decreased sexual drive, and less satisfaction post-treatment than physically healthy controls [30].

Using a constructivist grounded theory methodology, O'Callaghan and colleagues [31] found that interactions with music were helpful during and post-treatment for 12 AYAs to facilitate positive emotions and states, as well as transition off treatment.

\section{Social well-being}

Social well-being was reflected primarily in nine $(24 \%)$ studies and two secondarily. This section is defined by outcomes related to family distress, roles and relationships, affection/ sexual function, appearance, enjoyment, isolation, finances, education and employment.

AYA survivors reported that cancer worsened feelings of control over life, finances, work plans, social relationships, and relationship or family planning, and increased concern in these areas $[9,11,13]$. Trusting that they could find support when they needed it was reported as difficult $[9,29,38]$. AYAs described the need for greater peer and family support throughout their cancer journey [13,44], more opportunities to meet other AYA survivors [43], and the perception of inter-personal social support as promoting healthy psychosocial adjustment at various treatment stages $[40,45]$. Social support was found to be a significant 
predictor of psychosocial growth [25]. AYAs also endorsed a positive impact of cancer in the following areas: improved relationships, future planning/goals, and health competence [9].

Masculinity and self-image were impacted among testicular cancer survivors, who perceived the following social challenges: feeling different, viewing their differences as "damaged goods," struggles with cancer-related disclosures, and feelings of embarrassment that directly lead to treatment and medical care delays [12].

Overall, AYA's experiences transitioning off treatment are challenging, complex, and longterm [21, 27, 29]. A registry-based study found that $35 \%$ of survivors working or in school full-time pre-diagnosis believed that cancer had a negative impact on their plans, and greater than $50 \%$ reported problems with work or school post-diagnosis [34]. Acute Lymphocytic Leukemia (ALL) and Non-Hodgkin's Lymphoma survivors were less likely than other cancers to be working or in school 15 to 35 months post-diagnosis [34]. When examining time since treatment, AYAs greater than 2 years post-treatment were found to have greater adjustment in vocational environments compared to AYAs on active treatment [11].

After one or more years post-treatment for acute leukemia, no significant difference in psychosexual dysfunction was found between those treated with a bone marrow transplant (BMT) and chemotherapy only [30]. By gender, however, females overall reported decreased sexual drive, and female BMT survivors had lower frequency of sexual behaviors than all other groups.

\section{Survivorship care}

Survivorship care is comprised of essential components of care that facilitates access to comprehensive and coordinated care. The IOM has collaboratively defined this to include: prevention of relapse, new cancers or late effects, surveillance/follow-up care of cancer and assessment of medical and psychosocial late effects. This also includes intervention for consequences of cancer treatment, coordination between providers, health insurance access, and treatment-related informational needs. This section was primarily reflected in 14 (37\%) studies and 3 secondarily.

Survivorship, as defined by the National Cancer Institute, includes multifaceted aspects of the physical, psychosocial, and economic issues of cancer, beyond diagnosis and treatment [49]. Issues/concerns and specific needs for AYA survivorship emerged in 10 (26\%) of studies. Qualitative survivorship themes included: feelings about cancer survivorship, work/ education participation or outcomes, "being sick nor healthy," attitudes towards healthcare and intervention modalities, pain/fatigue, sexual functioning and fertility, risk behaviors (e.g., smoking, physical activity), and physical status and health [19, 24, 37, 40].

AYA information needs include information regarding aspects of survivorship that AYAs describe as important, helpful, or particularly lacking in the current healthcare system or survivorship culture. This includes needs at diagnosis, during treatment, and post-treatment. The content of these three areas of need include disease/treatment information and education regarding issues such as follow-up care, psychosocial resources, or fertility preservation. The primary theme noted in within this domain was the need for care and resources to be 
targeted to the age-specific and unique needs of the AYA population [45]. Numerous studies emphasized that age-appropriate information and affordable care are critical to providing long-term support to AYAs diagnosed with cancer [22, 42, 43, 53]. The need for flexibility in treatment scheduling to allow for work and family demands was frequently endorsed [35, 54]. A consistent theme was a desire to gain support from peer survivors or peer support groups, who were familiar with the unique challenges of managing cancer at a young age $[19,29,40]$. Informational needs regarding fertility were emphasized [29]. Results noted that many women felt they were insufficiently informed regarding health promotion, fertility concerns and fertility-related treatment effects; although they acknowledged the complexity of addressing this topic in the midst of survival and treatment-related concerns [17, 24, 29]. Older AYAs also described the need for care to foster greater autonomy in care decisions as they aged.

The importance of providing AYAs with relevant information as they transition to survivorship is consistent with the broader literature in cancer survivorship. AYAs desired information concerning possible late and long-term side-effects of treatment, their capacity to pursue family and work-related goals in the future, the transition to survivorship and what to expect, guidance on which health professionals to seek out for various issues, as well as access to supportive care to manage challenges in reintegrating to school and work roles [19, $26,34]$. A multi-center examination of this issue with a survey of 523 AYAs approximately 1 year from treatment for a variety of cancer diagnoses found that more than $50 \%$ reported unmet information needs, primarily around the potential for recurrence and secondary cancers, as well as long-term side effects [20]. Another study found that AYA survivors desired better educational and occupational support services after completing treatment [29]. Further analysis revealed that those who were male, older, non-white, of poorer overall health, with more treatment-related side effects (i.e., >3), and who perceived that their quality of medical care had been poorer were more likely to report unmet needs. Additionally, AYAs reporting poorer physical or mental health also reported greater unmet service and information needs. AYAs with more years elapsed since treatment also tended to report a worse attitude toward their ongoing medical care.

Health services and access to health services are defined generally as availability and access to interventions, medical and survivorship resources, medical appointments, screening, and/or follow-ups, as well as health-care utilization and psychosocial care needs for survivors and families. This additionally can include the delivery of medical information or services, and method interventions are conducted. Among AYAs, common health services and access themes included fertility preservation and information, cost and location barriers, insurance delays, lack of existing services, and communication with healthcare providers.

Overall, AYAs desire better communication regarding, and access to, fertility preservation options [23, 52, 53]. As noted above, some survivors perceived that such options were provided too late during their treatment, while others reported being provided inadequate information (or time) to make a sound decision, forcing them to make rushed treatment decisions, such as choosing between delayed treatment and infertility risks [17, 24]. A minority noted that providers did not discuss fertility options at all and felt they were not included in a decision that should have been their own [24]. AYAs that were provided with 
only written fertility information described it as not useful, or useful only to prompt dialogue with providers. Speaking with a fertility specialist was desirable, and women expressed desire to have open and honest discussions with providers regarding fertility [24].

Providers and patients agreed on the importance of having adequate health insurance and follow-up plans [43]. Availability of state-of-the-art, age-specific treatment and adequate health insurance were the most highly ranked healthcare needs among emerging adult survivors [52]. In a population-based study, survivors reported poorer overall health reported greater cost barriers. AYA survivors reported going without care as a result of higher costs than non-cancer controls, and cost barriers were particularly high for 20-29 year-old survivors, and for female survivors [23]. After completion of cancer treatment, survivors are often persistently faced with delayed treatment bills, high health insurance premiums, and long waits for qualify for life insurance due to having a "pre-existing condition" [21].

Post-treatment financial concerns can greatly affect AYA survivors' independence. As many AYAs must take time off from work or school for cancer care, they may lose insurance benefits and can experience a difficult time re-entering the workforce or re-enrolling in school. Because of treatment bills and continuing follow-up and healthcare costs, AYAs may become entirely financially dependent on their parents during the post-treatment phase [27, 53]. AYAs in their 20s or younger and/or less physically active AYAs were more likely to encounter cost barriers compared with older and/or more physically active AYAs.

Generally, studies reported that older AYAs were more likely to follow-up with oncological care on the recommended schedule. Similarly, females were generally more likely to comply with recommended oncological follow-up (but also experience greater cost barriers) compared to men. However, males were more likely to experience unmet service needs. Hispanic and non-Hispanic black survivors were less likely to participate in studies compared with other racial/ethnic groups, and non-white AYA survivors were more likely to experience unmet service needs. Additionally, geographic location also creates barriers for this population. For example, patients living in rural communities, compared to urban areas, have limited access to the specialized cancer care resources and services, often resulting in delayed diagnoses [28].

With respect to study quality, 6 of the 38 articles (16\%) satisfied all 9 indicators from the modified Downs and Black study quality checklist [46]. Eighteen of the included articles (47\%) were only missing 1 of the 9 quality indicators, with the vast majority of these studies not specifying whether the subjects who were prepared to participate were representative of the entire population from which they were recruited. A total of 13 articles (34\%) were missing 2 of the 9 quality indicators, with one remaining article missing 5 quality indicators [43].

\section{Discussion}

This review summarized topics across 38 published studies among AYAs diagnosed with cancer exclusively as an adolescent or young adult. Using existing QOL domains and recommendations from the Institute of Medicine (IOM), we classified our review based on 
four key areas: physical well-being and symptoms, psychological well-being, social wellbeing, and survivorship care. The range of presented quality of life concerns (physical, psychological, social) emphasize the AYAs' experience as described and reported as challenging, complex, and nuanced. Due to the unique developmental challenges of AYAs, this group is positioned to experience different outcomes from childhood or older adult survivor counterparts, and is underrepresented in the literature.

Survivorship care was represented in the largest number of studies, suggested the long-term and life-long impact on this group. These needs parallel the developmentally rich period of physical and psychological growth and autonomy that is normative for AYAs and coincides with their cancer diagnosis, treatment, and post-treatment survivorship. This growth involves both a family system (e.g., parental involvement during treatment) and may complicate or challenge the natural progression towards independence over time (e.g., financial independence, intimate relationships, autonomy over medical care).

AYAs require age-appropriate and flexible care and informational needs that garner autonomy geared towards long-term survivorship; these findings are supported by the IOM's workshop on AYAs with cancer (of note, this summary includes survivors of pediatric cancer as well) [48]. AYAs reported need for specific medical, psychosocial, and healthcare related information early during and post-treatment (e.g., fertility options, concerns). The importance of providing this information is an underemphasized area for AYAs, and is a potentially appropriate and necessary element of AYAs' growth and a sign of greater medical knowledge, recognition of the importance of appropriate follow-up care, and psychological growth. Findings suggest providers and families are cautious or shy away from delivering information about short- and long-term effects of treatment. However, some results were conflicting, such as findings that cancer negatively impacts social relationships, but may also strengthen parent and sibling relationships. Women demonstrated greater internalized mental health outcomes; greater psychosocial stress was found to increase likelihood of obtaining mental health treatment and complying with follow-up care. Obtaining mental health treatment and follow-up care were also found in other studies to improve outcomes. The gender difference highlights unique moderators and areas that can impact outcomes, such as greater physical activity, treatment type (i.e., bone marrow transplant, chemotherapy), or age at diagnosis. Solidifying potential moderators provides medical providers with points of entry for greater education, screening, and intervention. This may include providing AYA's with specific post-treatment exercise recommendations appropriate for their condition, or recommending body awareness approaches (e.g., yoga, Pilates, Tai Chi) or physical activity programs (e.g., First Descents).

\section{Limitations}

Numerous challenges exist for conducting research with this cohort, including small sample sizes, unknown selection biases (particularly for qualitative studies), self-report limitations (e.g., no ability to confirm diagnosis/treatment, lack of participants' knowledge of specific treatments received), limited generalizability, and lack of a comparison or control group. Acquiring parental consent and participants who are minors may deter institutions and IRB's away from establishing creative and innovative study recruitment and retention methods, 
relying on established registries or databases that often do not differentiate those diagnosed exclusively during AYA. Qualitative and large database analyses are limited by low minority representation and recruitment from rural or suburban cancer centers [18]. Overall, these limitations, along with AYAs' changing contact information (housing or employment transitions), preferred contact method (e.g., email, paper mail, landline, cell phone) and schedule for participation (accommodating school/college schedule), impact retention and participation. Results demonstrate a lack of longitudinal data with psychometrically validated measures to track outcome change; this overlooks time-sensitivity and its overlap with development during these ages [29]. Additionally, $16 \%$ of included studies reported outcomes among international AYA populations, which can limit generalizability because health care systems and access vary greatly internationally.

\section{Future directions}

At diagnosis and the start of treatment, healthcare providers and interventions should provide AYAs and families with greater resources about fertility (particularly for women), sexual functioning, late effects of treatment, social networks for survivors, disclosure, reentering school/work, and attaining revised educational/career goals. These issues are developmental and not static; they should be established and adjusted over time since treatment and AYAs' age. They should be reflected in follow-up care, survivorship care planning, continuing educational interventions (e.g., consultations, education pamphlets/ programs), and direct provider training. Future research should examine the effect of disruptions in educational, occupational, and family planning trajectories on QOL. Additionally, spirituality was examined in only one included study [26], and should be explored further among AYA survivors. In communities, educational or information-based workshops in high schools or college orientations may address the lack of understanding from AYAs' social networks, and ease challenges in disclosing.

Qualitative methodologies were used in $40 \%$ of these studies, highlighting a range of AYA concerns that have not yet been addressed in traditional psychological measures, thereby making validated quantitative measurement or screening difficult. As this literature broadens, more targeted quantitative work and interventions should be applied to specific outcomes found in these studies. A dearth of work has addressed AYA-specific interventions, as only 3 papers were intervention studies. $24 \%$ of included studies used population or registry-based surveys, highlighting feasibility for future researchers to use large databases to target specific outcomes among AYAs that can reach a larger community and diverse population.

To address AYAs' desire for more, rather than less, information, interventions and studies should explore what, when, and how much information minors and/or parents are provided, as well as the delivery and use of survivorship care plans. Findings suggest developing specific interventions targeting finances, relationships, body image issues, physical activity, and support to attain educational goals during and post-treatment. Interventions using social media or online components were found to be feasible and accessible to address these areas. Delivering care and interventions to AYAs from minority groups and living in rural areas is limited, delayed, and found to have negative impact on psychosocial outcomes. Additional 
barriers to utilizing interventions and study methodology should be explored. Furthermore, identifying barriers to accessing healthcare services during and post-treatment provides direction for providers and families to effectively advocate for their specific healthcare. This is a unique population that engenders multifaceted variables contributing to post-treatment outcomes. As such, they require equally multifaceted care and consideration in research and clinical practice.

\section{Acknowledgments}

The authors wish to acknowledge Dr. Jamie Ostroff for her mentorship and guidance for this manuscript.

Funding This research was supported by a National Institutes of Health Research Training Grant (T32 CA009461-25), as well as a National Institutes of Health Support Grant (P30 CA08748-49), which provides partial support for the Behavioral Research Methods Core Facility used in conducting this investigation.

\section{Appendix}

\section{Table 2}

Complete list of medical subject heading (MeSH) and keyword terms

\begin{tabular}{|c|c|c|}
\hline Search & Query & $\begin{array}{l}\text { Items } \\
\text { found }\end{array}$ \\
\hline \#4 & Search \#1)AND\#2)AND\#3 & 16,493 \\
\hline \#3 & $\begin{array}{l}\text { Search [outcome OR “Outcome and Process } \\
\text { Assessment (Health Care)" MeSH) OR outcomes OR } \\
\text { result OR results OR adaptation OR } \\
\text { "Adaptation, Psychological" (MeSH) OR } \\
\text { "Acclimatization" } \\
\text { (MeSH) OR adjustment OR "Social Adjustment" } \\
\text { (MeSH) OR distress OR "quality of life" OR "Quality of } \\
\text { Life" (MeSH) OR } \\
\text { QOL OR coping OR "unmet needs" OR "Needs } \\
\text { Assessment" (MeSH)] Sort by: PublicationDate }\end{array}$ & $6,417,999$ \\
\hline \#2 & $\begin{array}{l}\text { Search [psychology OR "Psychology" (MeSH) OR } \\
\text { psychological OR psychosocial OR "Psychotherapy" } \\
\text { (MeSH) OR psychiatry } \\
\text { OR "Psychiatry" MeSH) OR psychiatric OR support OR } \\
\text { "Social Support" (MeSH) OR behavior OR "Behavior } \\
\text { Therapy" (MeSH) OR "behavioral” Or "cognitive } \\
\text { behavioural" OR CBT } \\
\text { OR talk OR integrative OR "Integrative" (MeSH) OR } \\
\text { alternative OR "Complementary Therapies" (MeSH) OR } \\
\text { breathing OR meditation OR family OR } \\
\text { psychopharmacology OR psychotropic } \\
\text { OR "Psychotropic Drugs"(MeSH) AND (intervention } \\
\text { OR "Intervention Studies" MeSH) OR treatment OR } \\
\text { "Therapeutics" (MeSH)] Sort by: PublicationDate }\end{array}$ & $3,096,896$ \\
\hline$\# 1$ & $\begin{array}{l}\text { Search [adolescent OR “Adolescent” (MeSH) OR “young } \\
\text { adult” OR "Young Adult" (MeSH) AND (neoplasm OR } \\
\text { "neoplasms" (MeSH) OR neoplasms OR malignan* OR } \\
\text { sarcoma OR sarcomas } \\
\text { OR carcinoma OR carcinomas OR tumor OR tumors OR } \\
\text { tumour OR tumours OR lesion OR lesions OR cancer } \\
\text { OR neoplasia) AND (survivor OR "Survivors" (MeSH) } \\
\text { OR survivors OR survivorship)] } \\
\text { Sort by: Publication Date }\end{array}$ & 43,473 \\
\hline
\end{tabular}

\section{References}

1. Bleyer A, Barr R, Hayes-Lattin B, et al. The distinctive biology of cancer in adolescents and young adults. Nat Rev Cancer. 2008; 8:288-98. [PubMed: 18354417] 
2. Institute of Medicine. Identifying and addressing the needs of adolescents and young adults with cancer: workshop summary. The National Academies Press; Washington, DC: 2013.

3. Bleyer, A.; O'Leary, M.; Barr, R.; Ries, LAG. Cancer epidemiology in older adolescents and young adults 15 to 29 years of age, including SEER incidence and survival: 1975-2000. National Cancer Institute; Bethesda: 2006. NIH Pub. No. 06-5767

4. Bleyer WA. Cancer in older adolescents and young adults: epidemiology, diagnosis, treatment, survival, and importance of clinical trials. Med Pediatr Oncol. 2002; 38:1-10. [PubMed: 11835231]

5. Hewitt, M.; Greenfield, S.; Stovall, S., editors. From cancer patient to cancer survivor: lost in translation. The National Academies Press; Washington, DC: 2005.

6. Albritton K, Bleyer WA. The management of cancer in the older adolescent. Eur J Cancer. 2003; 39:2584-99. [PubMed: 14642921]

7. Evan EE, Zeltzer LK. Psychosocial dimensions of cancer in adolescents and young adults. Cancer. 2006; 107:1663-71. [PubMed: 16921479]

8. Badr H, Chandra J, Paxton RJ, et al. Health-related quality of life, lifestyle behaviors, and intervention preferences of survivors of childhood cancer. J Cancer Surviv Res Pract. 2013; 7:52334.

9. Bellizzi KM, Smith A, Schmidt S, et al. Positive and negative psychosocial impact of being diagnosed with cancer as an adolescent or young adult. Cancer. 2012; 118:5155-62. [PubMed: 22415815]

10. Bloom JR, Stewart SL, D'Onofrio CN, Luce J, Banks PJ. Addressing the needs of young breast cancer survivors at the 5 year milestone: can a short-term, low intensity intervention produce change? J Cancer Surviv Res Pract. 2008; 2:190-204.

11. Carpenter PJ, Morrow GR, Schmale AH. The psychosocial status of cancer patients after cessation of treatment. J Psychosoc Oncol. 1989; 7:95-103.

12. Carpentier MY, Fortenberry JD, Ott MA, Brames MJ, Einhorn LH. Perceptions of masculinity and self-image in adolescent and young adult testicular cancer survivors: implications for romantic and sexual relationships. Psychooncology. 2011; 20:738-45. [PubMed: 20878864]

13. D'Agostino NM, Edelstein K. Psychosocial challenges and resource needs of young adult cancer survivors: implications for program development. J Psychosoc Oncol. 2013; 31:585-600. [PubMed: 24175897]

14. Deyell RJ, Lorenzi M, Ma S, et al. Antidepressant use among survivors of childhood, adolescent and young adult cancer: a report of the childhood, adolescent and young adult cancer survivor (CAYACS) research program. Pediatr Blood Cancer. 2013; 60:816-22. [PubMed: 23281214]

15. Dieluweit U, Seitz DCM, Besier T, et al. Utilization of psychosocial care and oncological followup assessments among German long-term survivors of cancer with onset during adolescence. Klin Padiatr. 2011; 223:152-8. [PubMed: 21462102]

16. Dyson GJ, Thompson K, Palmer S, Thomas DM, Schofield P. The relationship between unmet needs and distress amongst young people with cancer. Support Care Cancer. 2012; 20:75-85. [PubMed: 21311915]

17. Gorman JR, Usita PM, Madlensky L, Pierce JP. Young breast cancer survivors: their perspectives on treatment decisions and fertility concerns. Cancer Nurs. 2011; 34:32-40. [PubMed: 20697269]

18. Harlan LC, Lynch CF, Keegan THM, et al. Recruitment and follow-up of adolescent and young adult cancer survivors: the AYA HOPE Study. J Cancer Surviv. 2011; 5:305-14. [PubMed: 21274648]

19. Hauken M, Larsen TMB, Holsen I. Meeting reality: young adult cancer survivors' experiences of reentering everyday life after cancer treatment. Cancer Nurs. 2013; 36:E17-26. [PubMed: 23348661]

20. Keegan THM, Lichtensztajn DY, Kato I, et al. Unmet adolescent and young adult cancer survivors information and service needs: a population-based cancer registry study. J Cancer Surviv Res Pract. 2012; 6:239-50.

21. Keim-Malpass J, Steeves RH. Talking with death at a diner: young women's online narratives of cancer. Oncol Nurs Forum. 2012; 39:373-8. 406. [PubMed: 22750895]

22. Keim-Malpass J, Baernholdt M, Erickson JM, et al. Blogging through cancer young women's persistent problems shared online. Cancer Nurs. 2013; 36:163-72. [PubMed: 22495504] 
23. Kirchhoff AC, Lyles CR, Fluchel M, Wright J, Leisenring W. Limitations in health care access and utilization among long-term survivors of adolescent and young adult cancer. Cancer. 2012; 118:5964-72. [PubMed: 23007632]

24. Lee RJ, Wakefield A, Foy S, et al. Facilitating reproductive choices: the impact of health services on the experiences of young women with breast cancer. Psychooncology. 2011; 20:1044-52. [PubMed: 20818600]

25. Love C, Sabiston CM. Exploring the links between physical activity and posttraumatic growth in young adult cancer survivors. Psychooncology. 2011; 20:278-86. [PubMed: 20238374]

26. Mattson MR, Demshar RK, Daly BJ. Quality of life of young adult survivors of hematologic malignancies. Cancer Nurs. 2013; 36:E1-7. [PubMed: 22293158]

27. Miedema B, Hamilton R, Easley J. From "invincibility" to "normalcy": coping strategies of young adults during the cancer journey. Palliat Support Care. 2007; 5:41-9. [PubMed: 17461370]

28. Miedema B, Easley JK, Robinson LM. Comparing urban and rural young adult cancer survivors' experiences: a qualitative study. Rural Remote Health. 2013; 13:2324. [PubMed: 23534891]

29. Millar B, Patterson P, Desille N. Emerging adulthood and cancer: how unmet needs vary with timesince-treatment. Palliat Support Care. 2010; 8:151-8. [PubMed: 20307366]

30. Mumma GH, Mashberg D, Lesko LM. Long-term psychosexual adjustment of acute leukemia survivors: impact of marrow transplantation versus conventional chemotherapy. Gen Hosp Psychiatry. 1992; 14:43-55. [PubMed: 1730401]

31. O'Callaghan C, Barry P, Thompson K. Music's relevance for adolescents and young adults with cancer: a constructivist research approach. Support Care Cancer. 2012; 20:687-97. [PubMed: 21311914]

32. Park CL, Cho D, Wortmann JH. The impact of yoga upon young adult cancer survivors. Complement Ther Clin Pract. 2013; 19:77-82. [PubMed: 23561064]

33. Parsons JA, Eakin JM, Bell RS, Franche RL, Davis AM. “So, are you back to work yet?” Reconceptualizing 'work' and 'return to work' in the context of primary bone cancer. Soc Sci Med. 2008; 67:1826-36. [PubMed: 18851893]

34. Parsons HM, Harlan LC, Lynch CF, et al. Impact of cancer on work and education among adolescent and young adult cancer survivors. J Clin Oncol. 2012; 30:2393-400. [PubMed: 22614977]

35. Phillips-Salimi CR, Andrykowski MA. Physical and mental health status of female adolescent/ young adult survivors of breast and gynecological cancer: a national, population-based, casecontrol study. Support Care Cancer. 2013; 21:1597-604. 1-8. [PubMed: 23306935]

36. Rabin C, Simpson N, Morrow K, Pinto B. Behavioral and psychosocial program needs of young adult cancer survivors. Qual Health Res. 2011; 21:796-806. [PubMed: 20705863]

37. Rabin C, Simpson N, Morrow K, Pinto B. Intervention format and delivery preferences among young adult cancer survivors. Int J Behav Med. 2013; 20:304-10. [PubMed: 22328444]

38. Sammallahti P, Lehto-Salo P, Maenpaa H, Elomaa I, Aalberg V. Psychological defenses of young osteosarcoma survivors. Psychooncology. 1995; 4:283-6.

39. Tai E, Buchanan N, Townsend J, et al. Health status of adolescent and young adult cancer survivors. Pediatr Blood Cancer. 2012; 58:1066.

40. Thompson K, Palmer S, Dyson G. Adolescents \& young adults: issues in transition from active therapy into follow-up care. Eur J Oncol Nurs. 2009; 13:207-12. [PubMed: 19539526]

41. Valle CG, Tate DF, Mayer DK, Allicock M, Cai J. A randomized trial of a Facebook-based physical activity intervention for young adult cancer survivors. J Cancer Surviv Res Pract. 2013

42. Yanez B, Garcia SF, Victorson D, Salsman JM. Distress among young adult cancer survivors: a cohort study. Support Care Cancer. 2013; 21:2403-8. [PubMed: 23568764]

43. Zebrack B, Bleyer A, Albritton K, Medearis S, Tang J. Assessing the health care needs of adolescent and young adult cancer patients and survivors. Cancer. 2006; 107:2915-23. [PubMed: 17103383]

44. Zebrack BJ, Mills J, Weitzman TS. Health and supportive care needs of young adult cancer patients and survivors. J Cancer Surviv. 2007; 1:137-45. [PubMed: 18648954] 
45. Zebrack B, Chesler MA, Kaplan S. To foster healing among adolescents and young adults with cancer: what helps? What hurts? Support Care Cancer. 2010; 18:131-5. [PubMed: 19690897]

46. Downs SH, Black N. The feasibility of creating a checklist for the assessment of the methodological quality both of randomised and non-randomised studies of health care interventions. J Epidemiol Community Health. 1998; 52:377-84. [PubMed: 9764259]

47. Senn SJ. Overstating the evidence: double counting in meta-analysis and related problems. BMC Med Res Methodol. 2009; 9:10. [PubMed: 19216779]

48. Nass SJ, Beaupin LK, Demark-Wahnefried W, et al. Identifying and addressing the needs of adolescents and young adults with cancer: summary of an Institute of Medicine workshop. Oncologist. 2015; 20:186-95. [PubMed: 25568146]

49. NCI. Survivorship. National Cancer Institute at the National Institutes of Health; Available from: http://www.cancer.gov/about-cancer/coping/survivorship [Accessed 1 Feb 2016]

50. Cassileth BR, Keefe FJ. Integrative and behavioral approaches to the treatment of cancer-related neuropathic pain. Oncologist. 2010; 15(Suppl 2):19-23. [PubMed: 20489193]

51. Cassileth, BR. The complete guide to complementary therapies in cancer care. WSPC; Singapore: 2011.

52. Zebrack BJ, Mills J, Weitzman TS. Health and supportive care needs of young adult cancer patients and survivors. J Cancer Surviv Res Pract. 2007; 1:137-45.

53. Miedema B, Easley J, Robinson LM. Do current cancer follow-up care practices meet the needs of young adult cancer survivors in Canada? A qualitative inquiry. Curr Oncol. 2013; 20:14-22. [PubMed: 23443642]

54. Rabin C, Simpson N, Morrow K, Pinto B. Intervention format and delivery preferences among young adult cancer survivors. Int J Behav Med. 2013; 20:304-10. [PubMed: 22328444] 


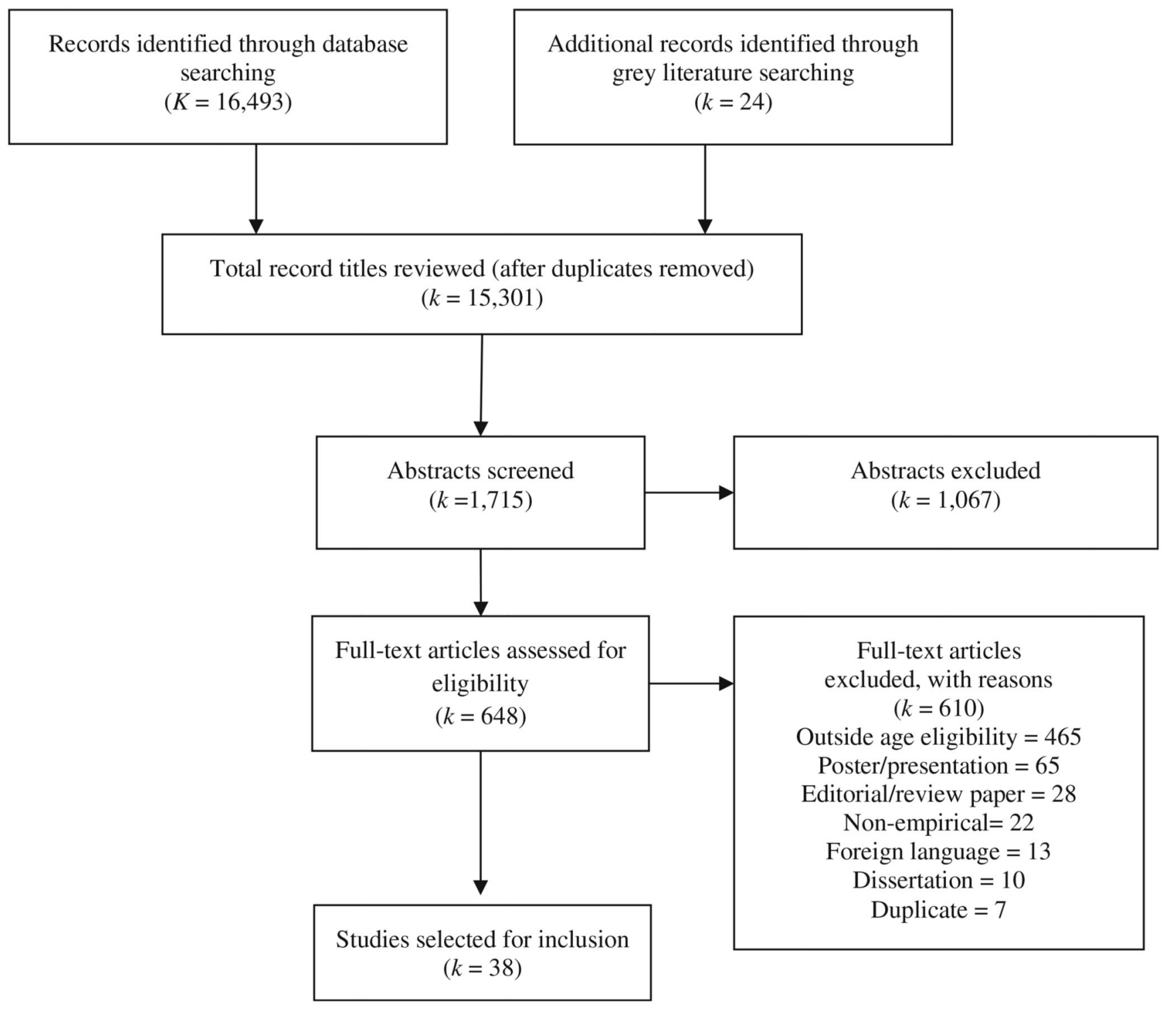

Fig. 1.

PRISMA flow chart 


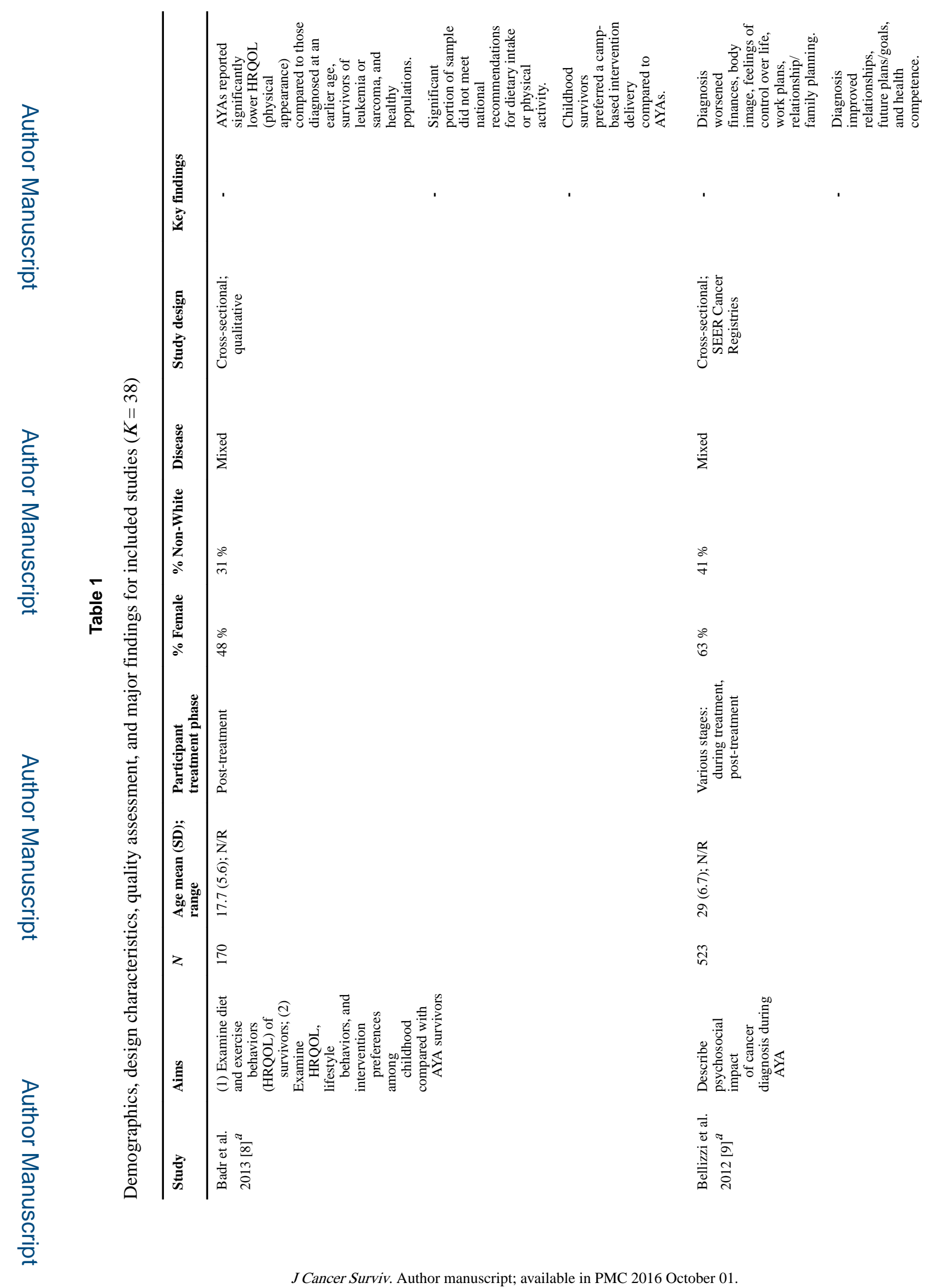




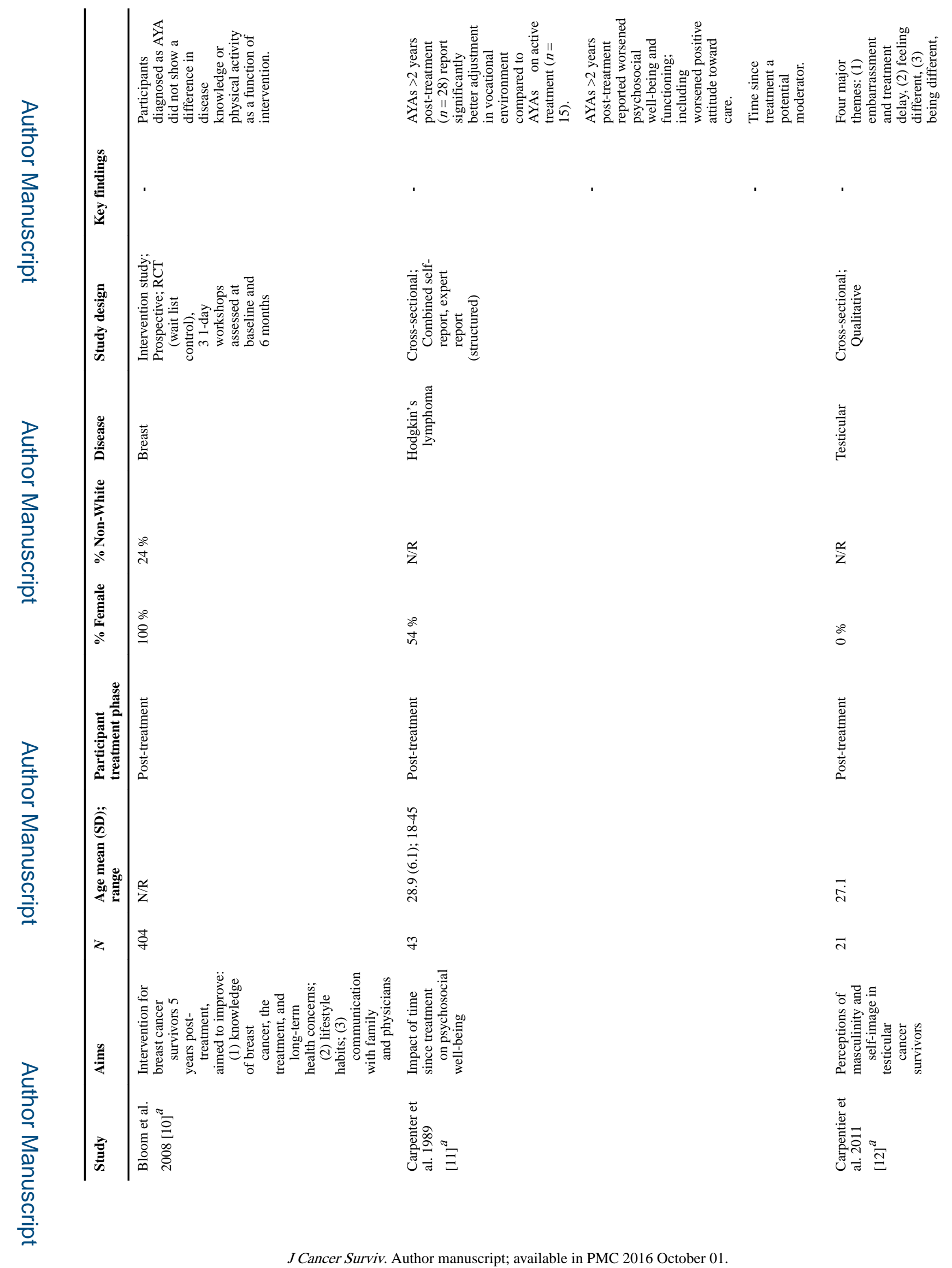




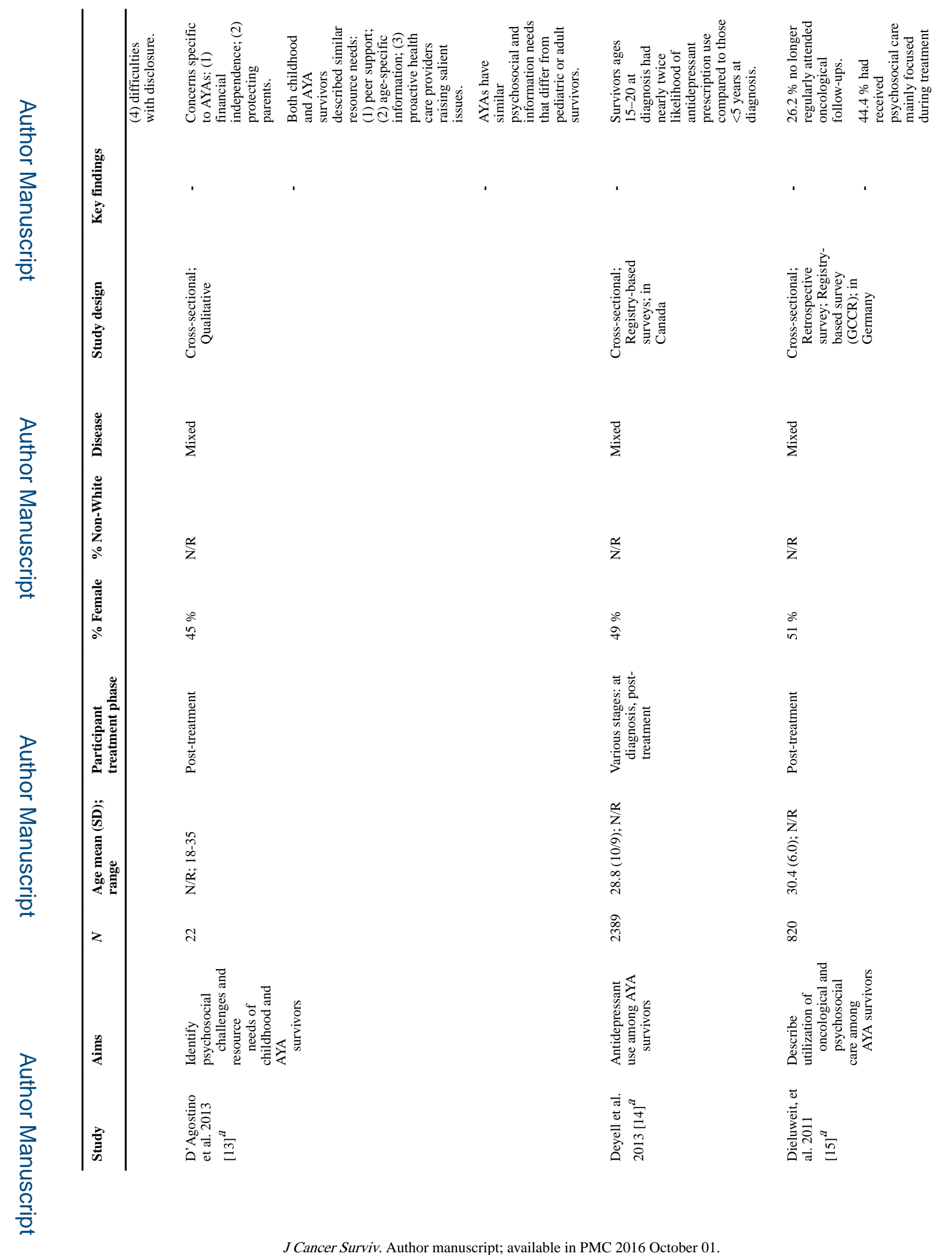




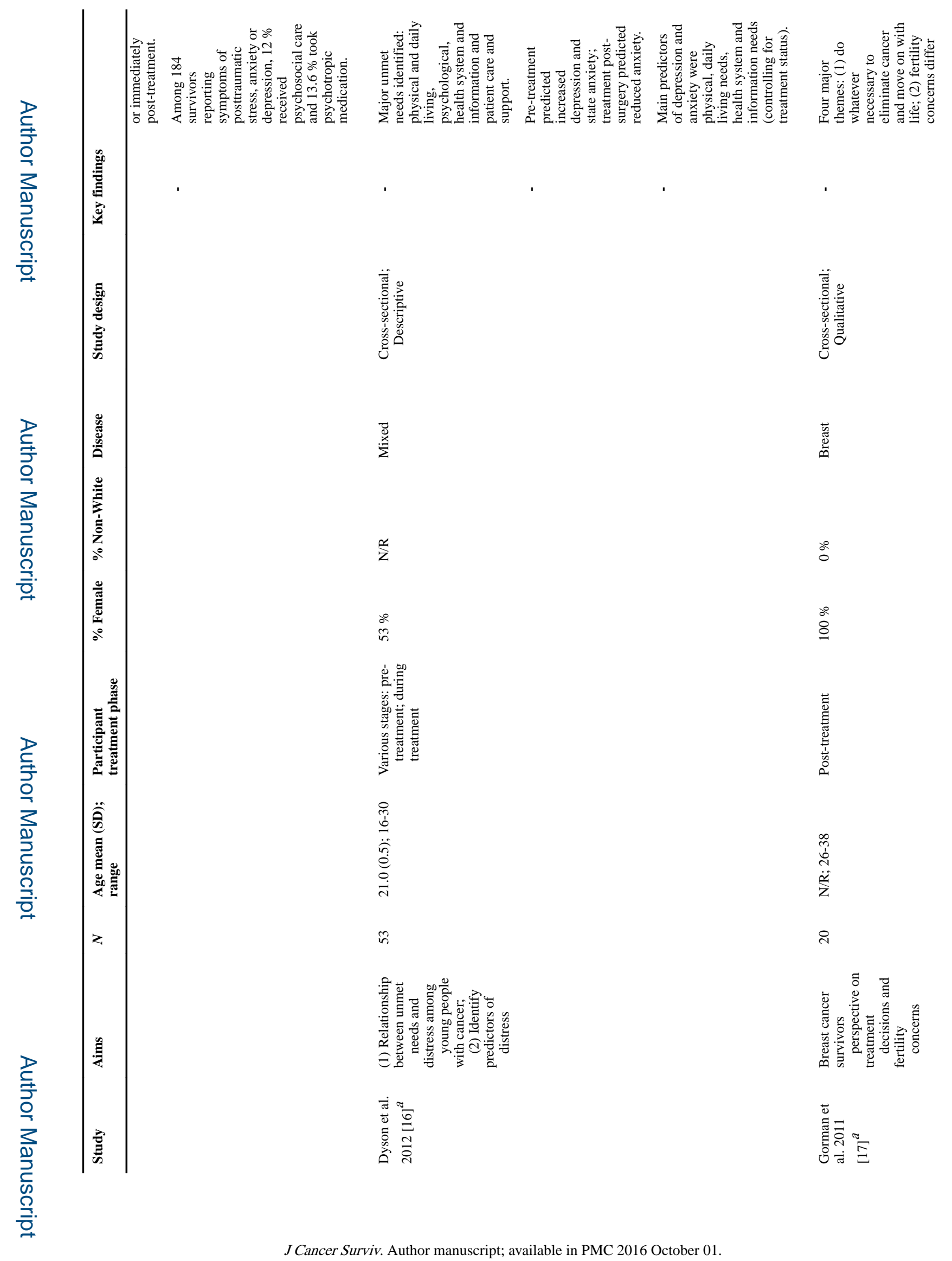




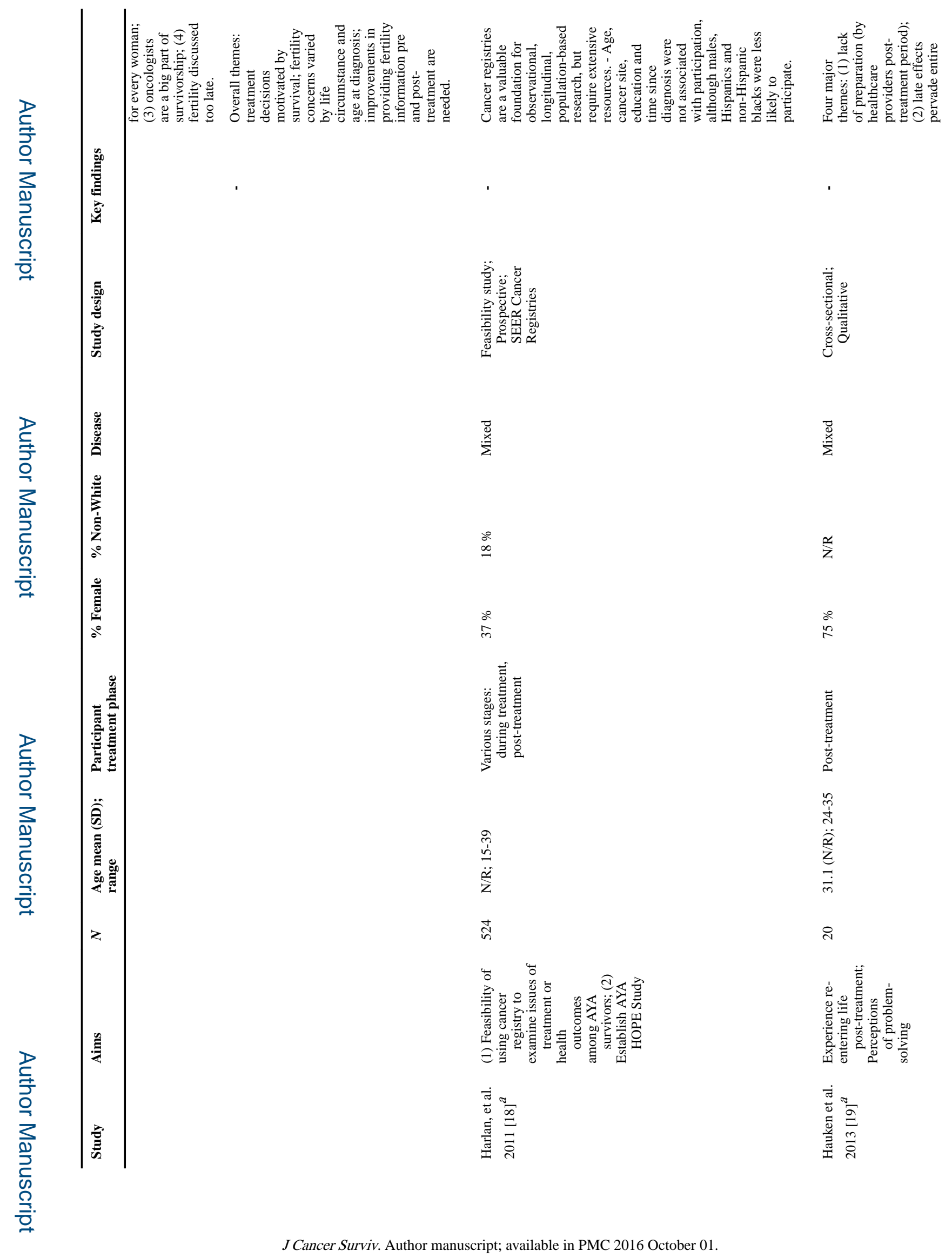




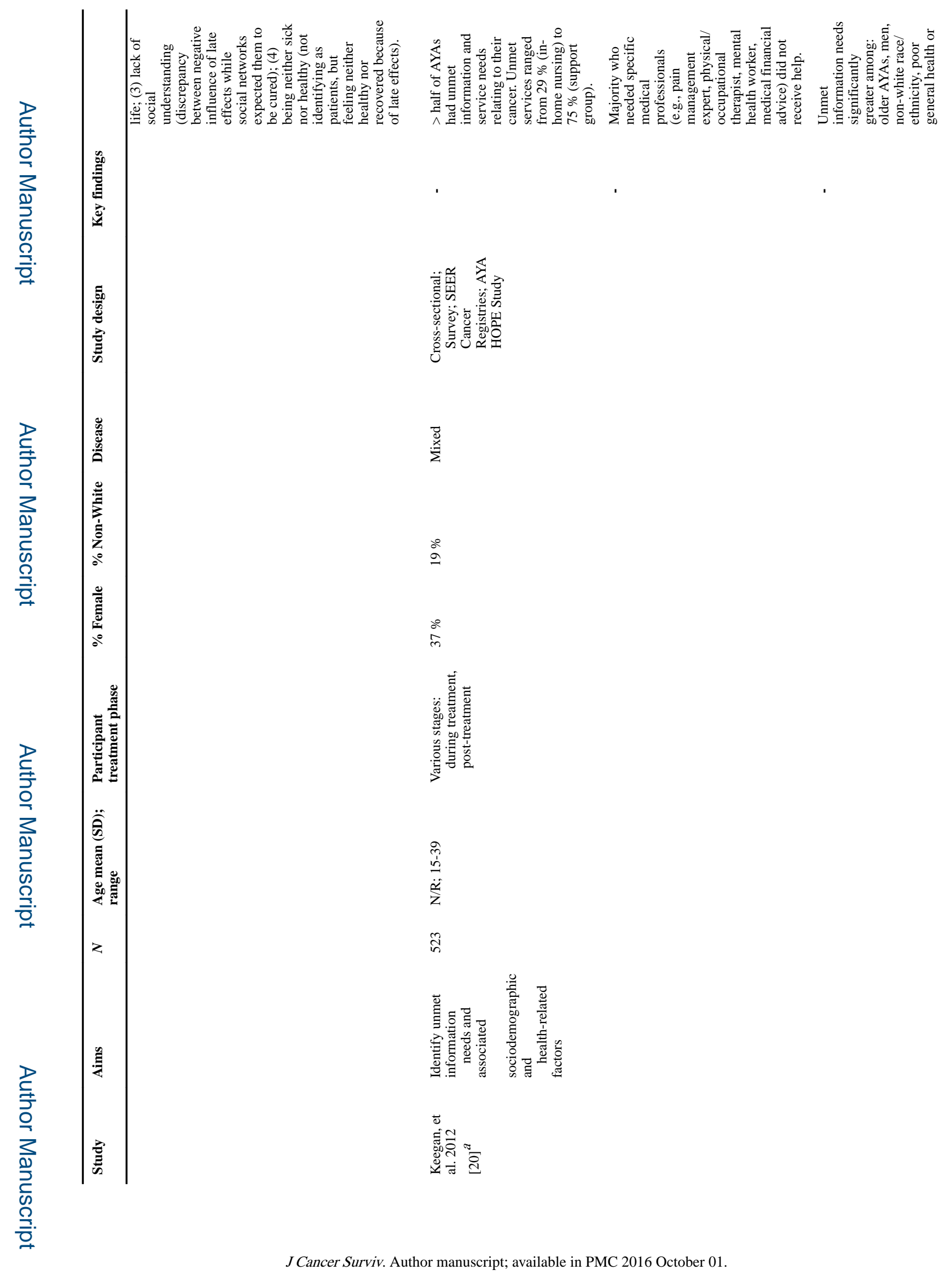




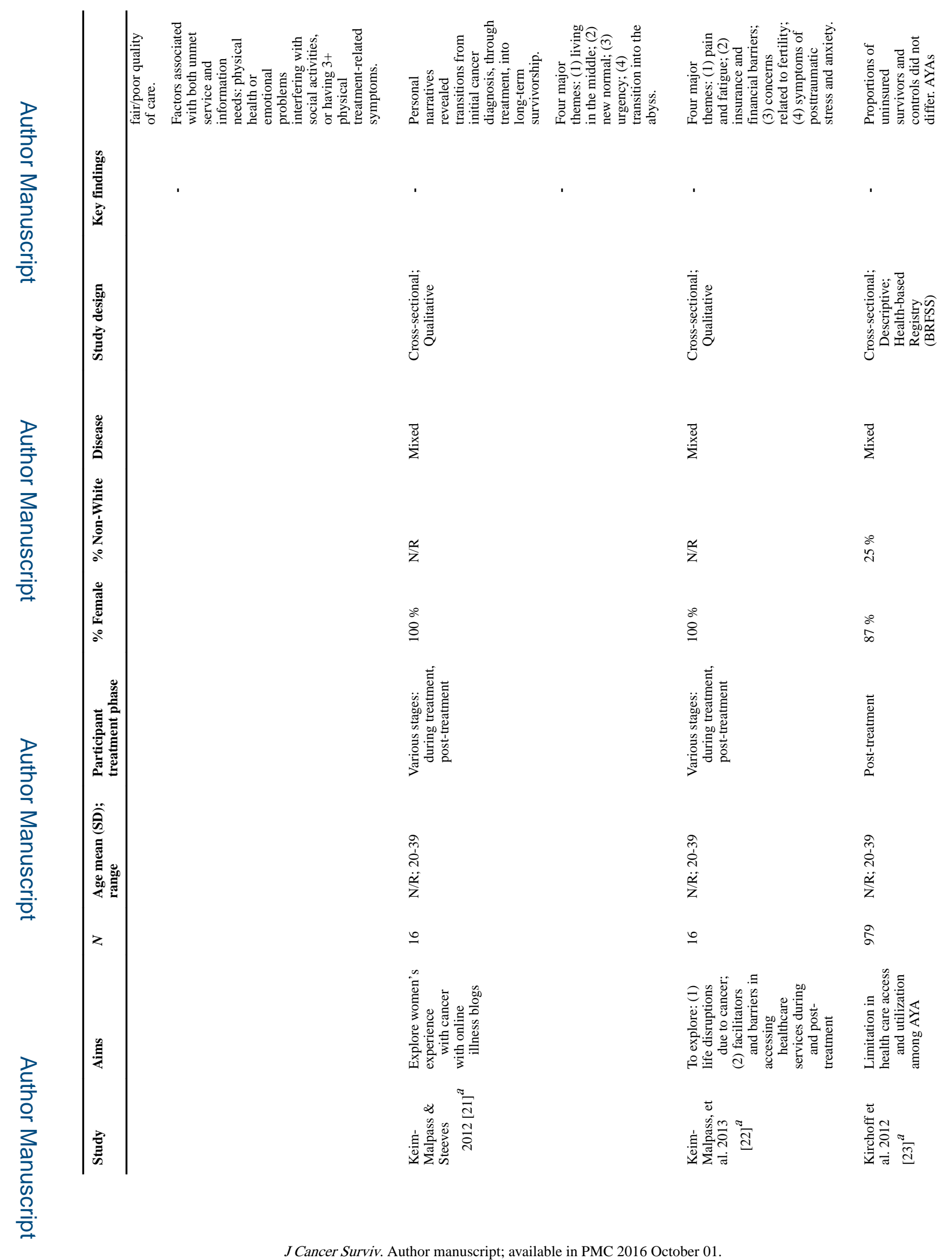




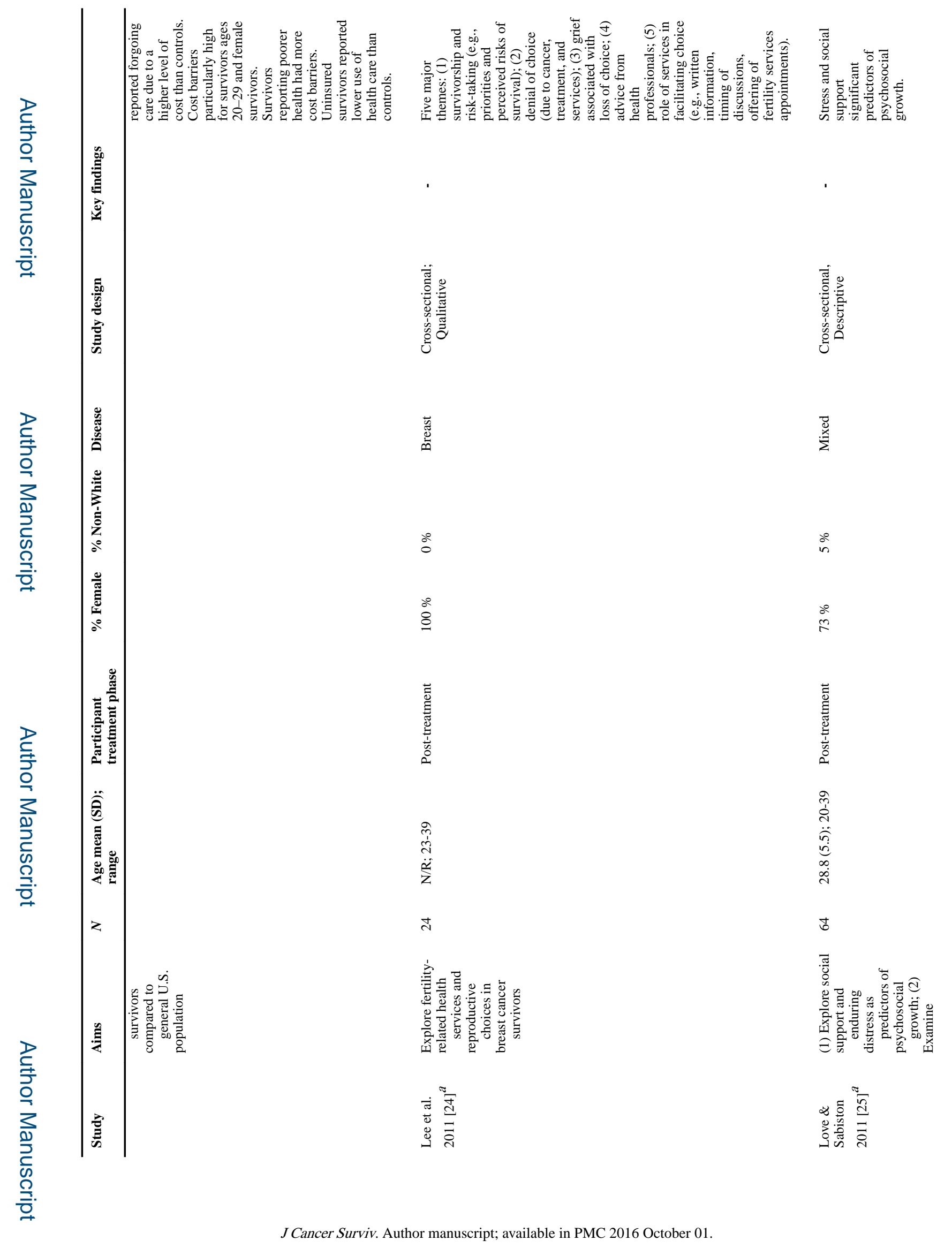




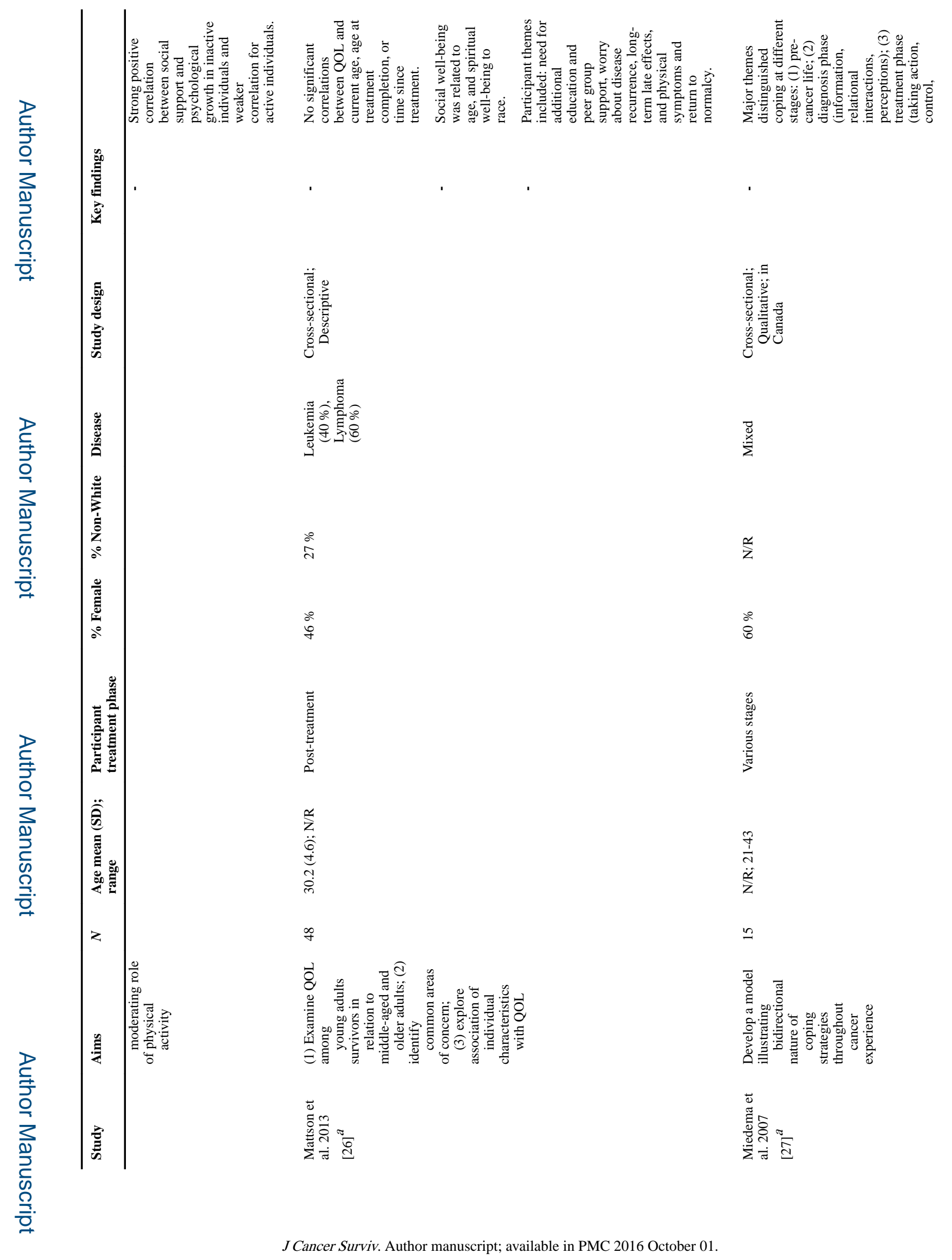




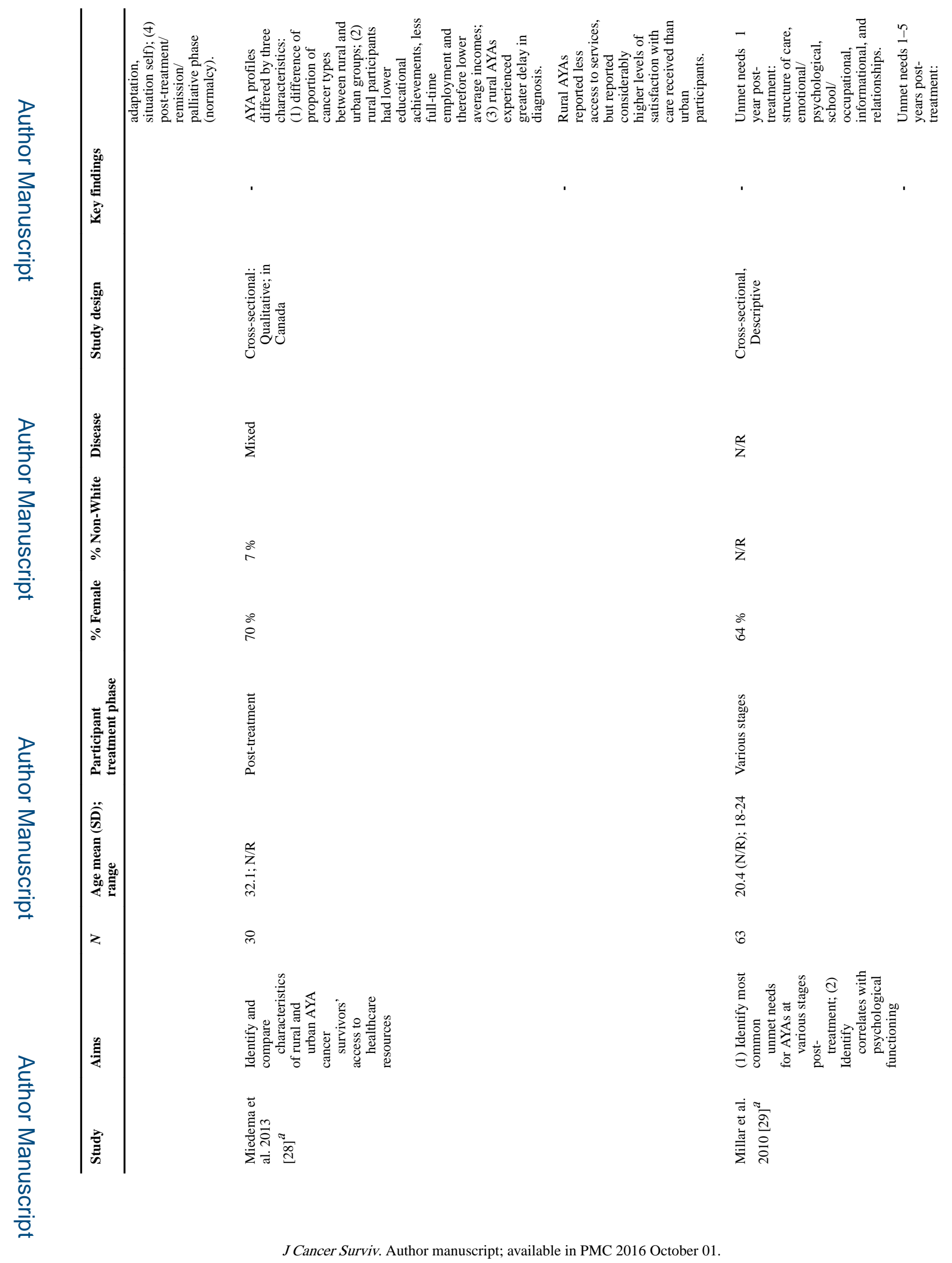




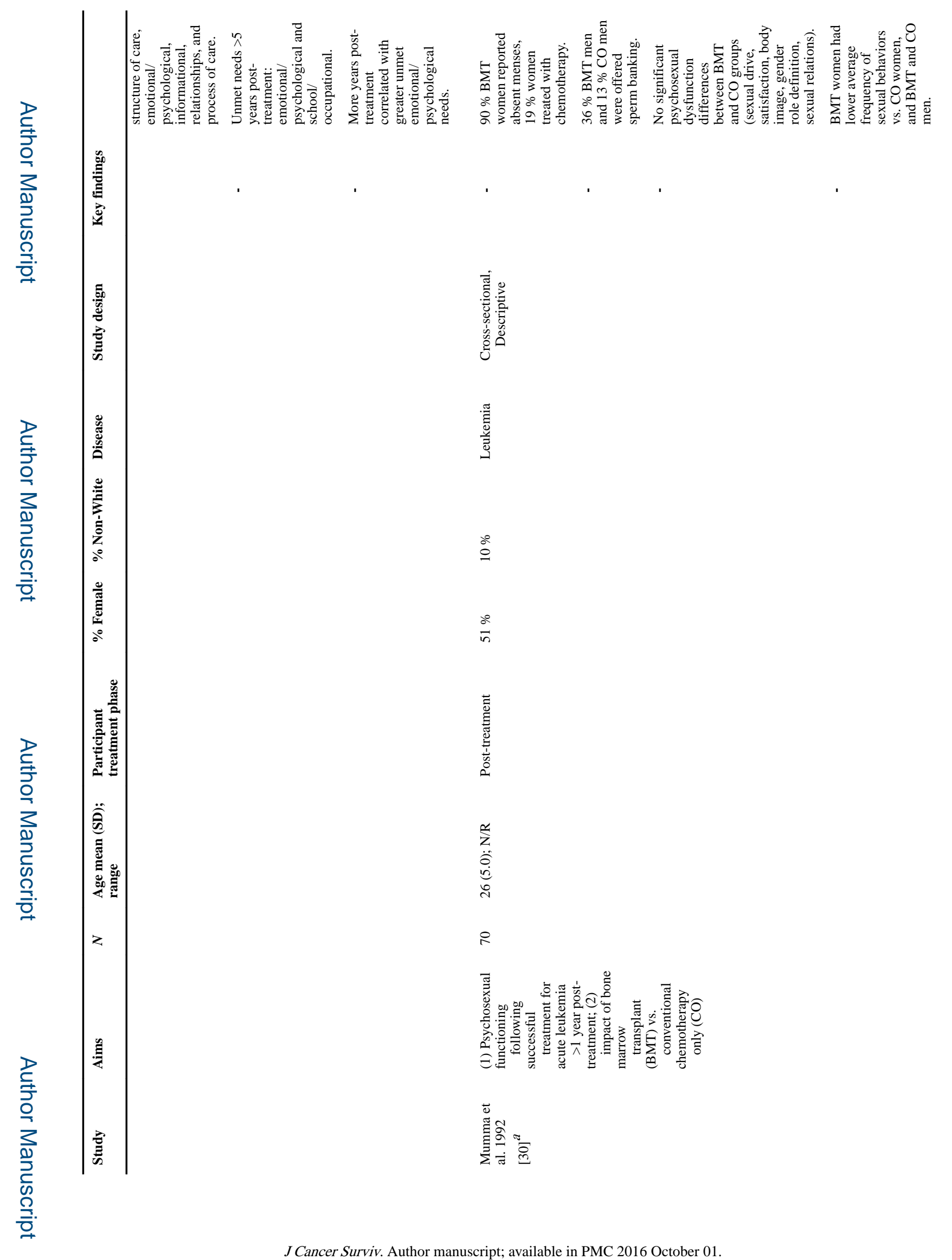




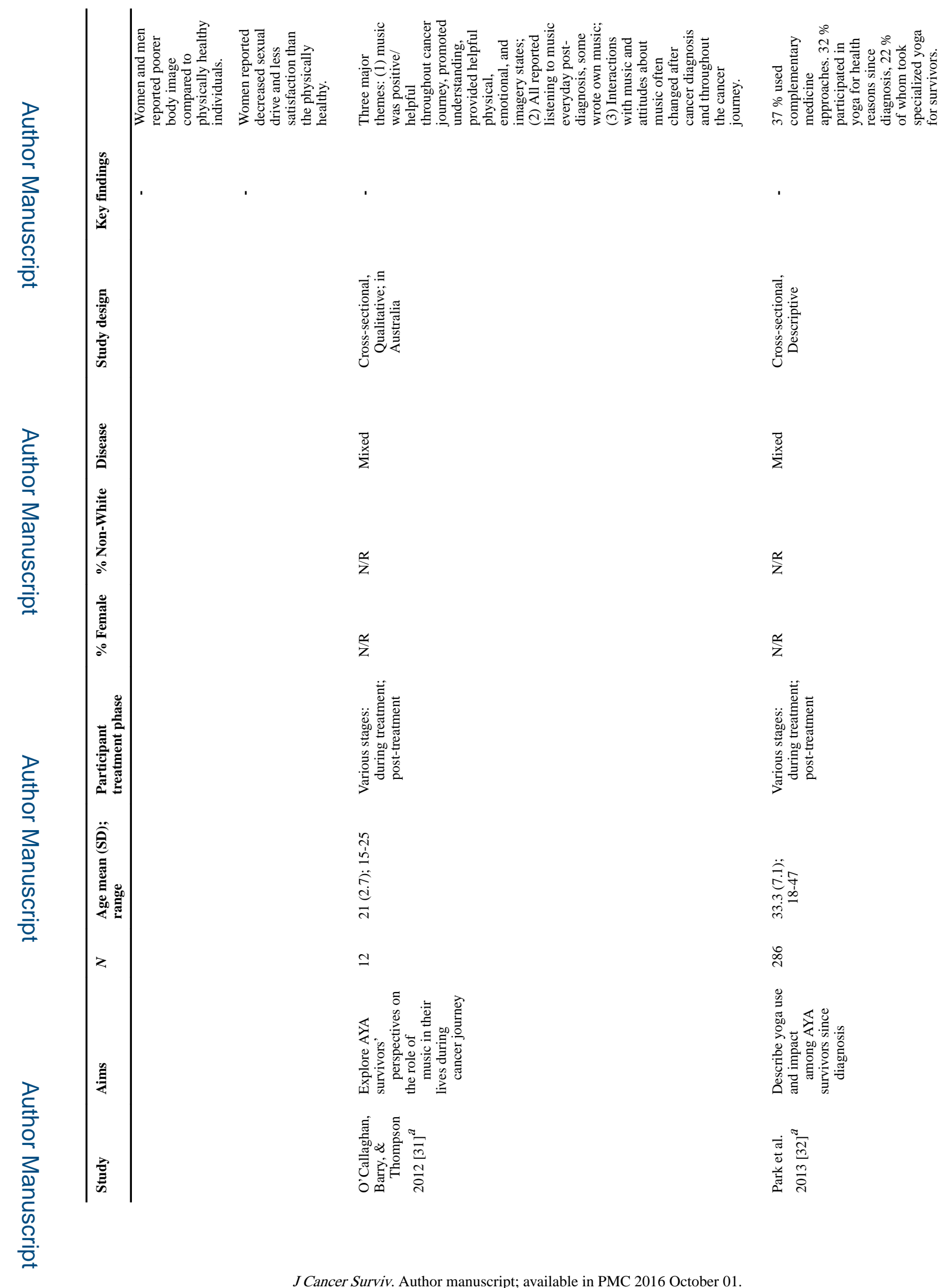




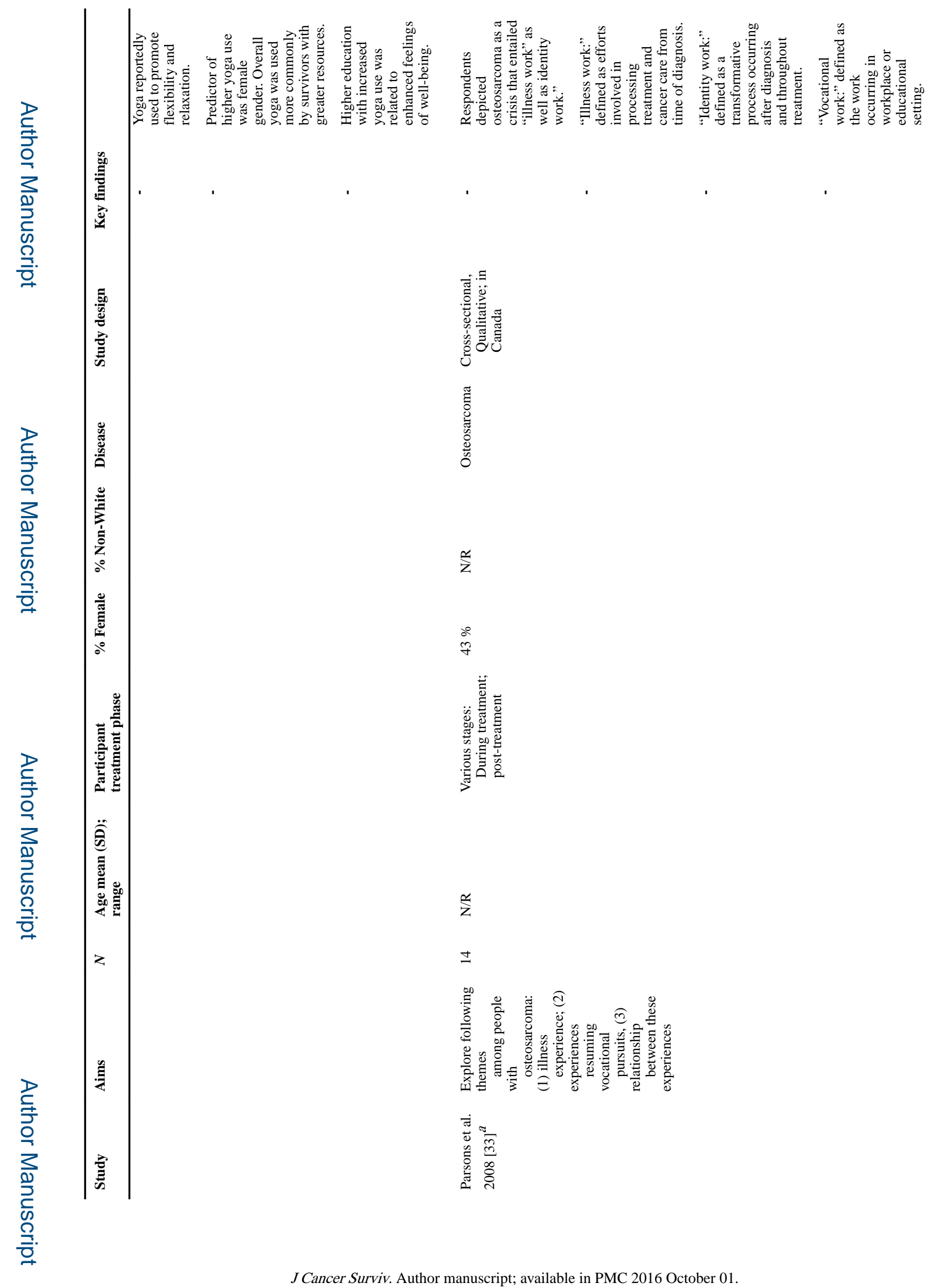


Barnett et al.

Page 31

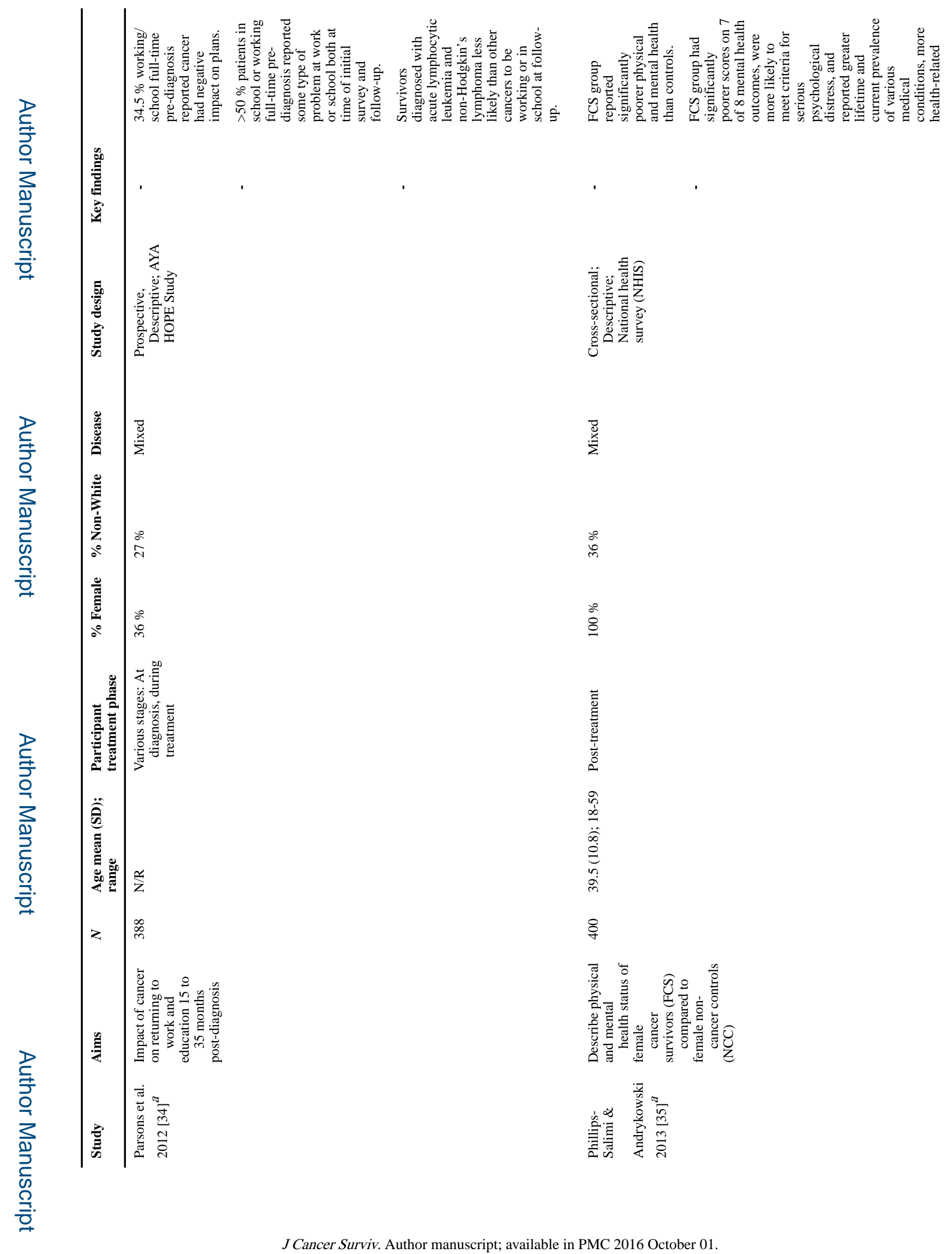


Barnett et al.

Page 32

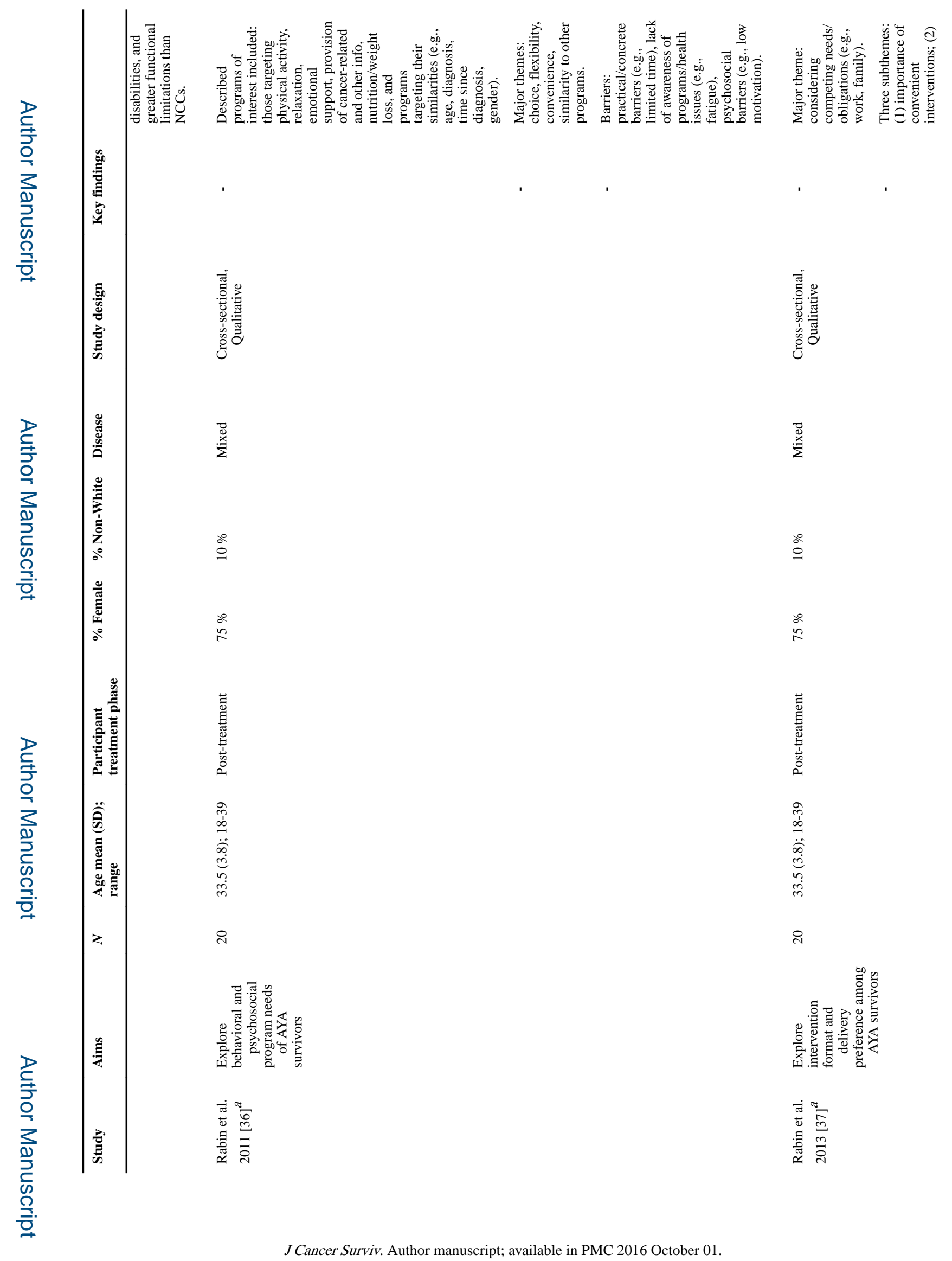




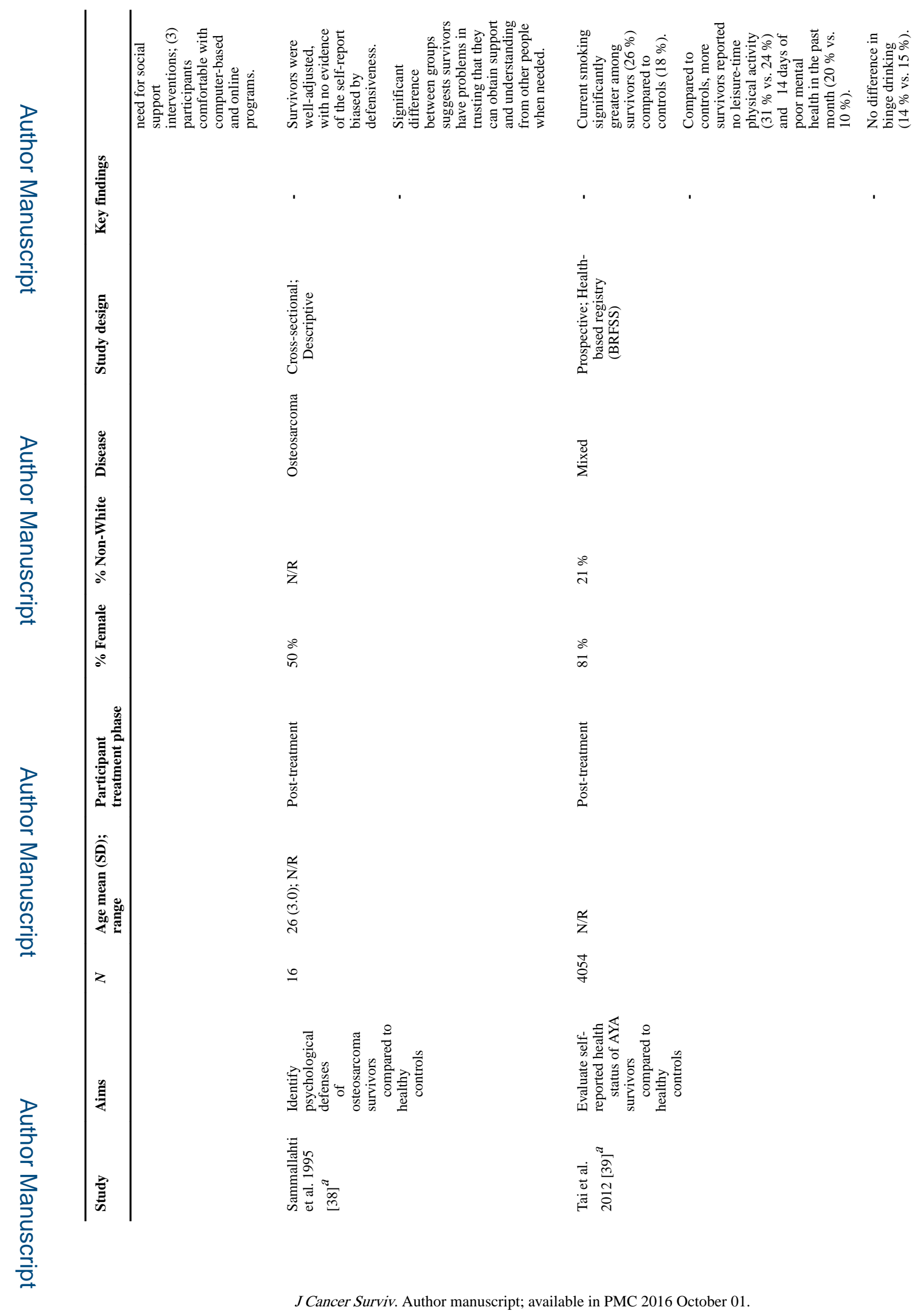




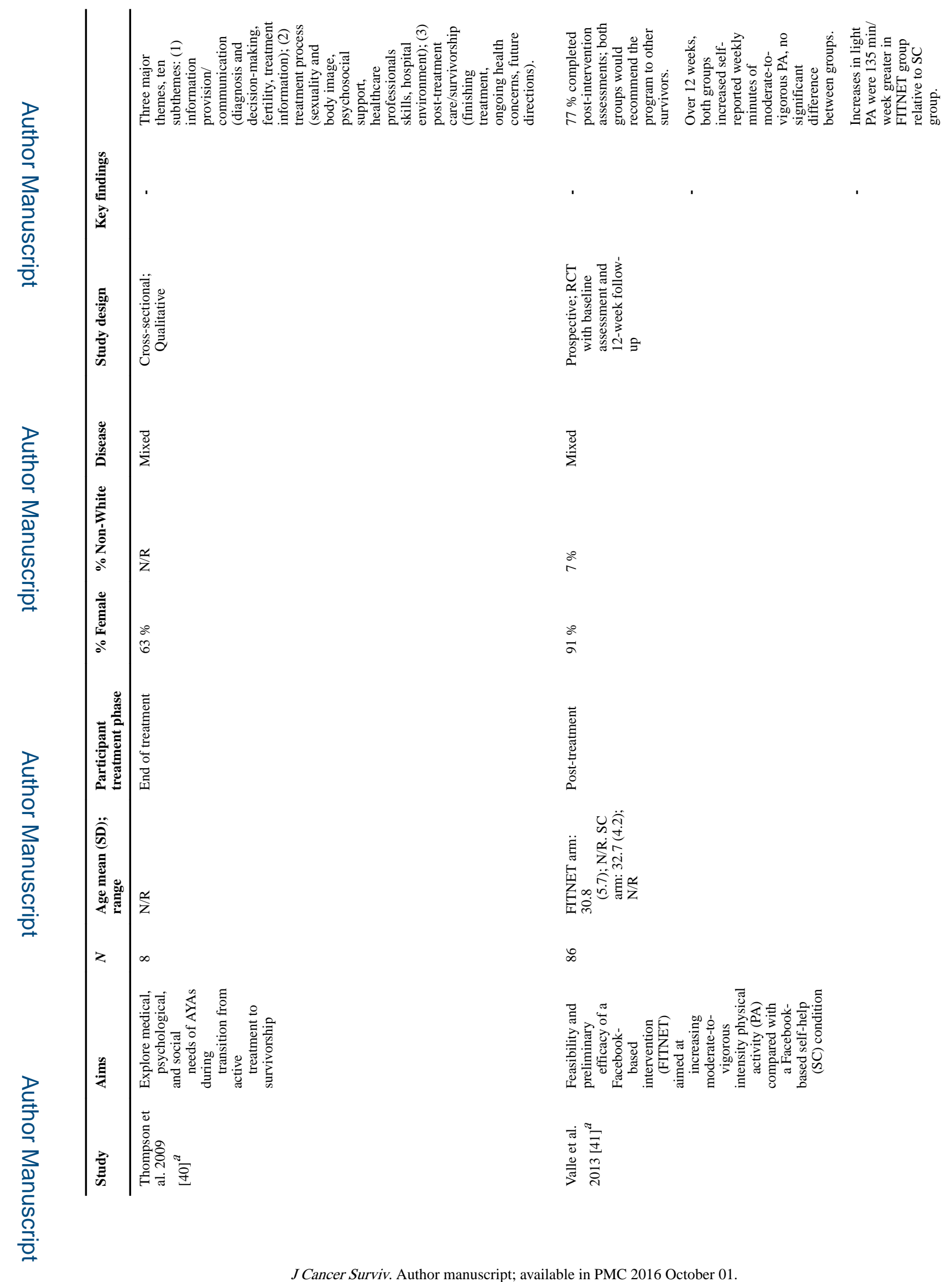




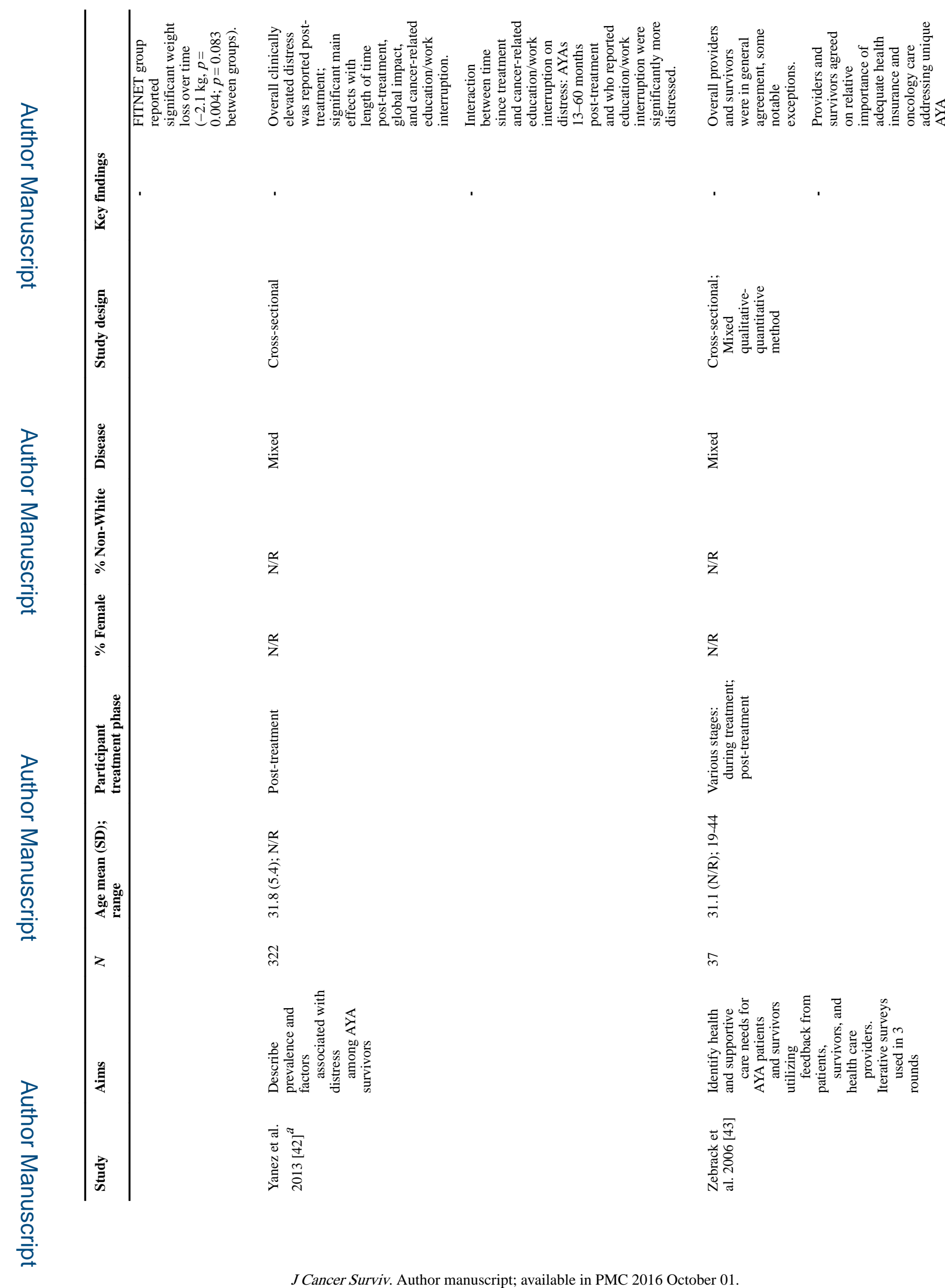




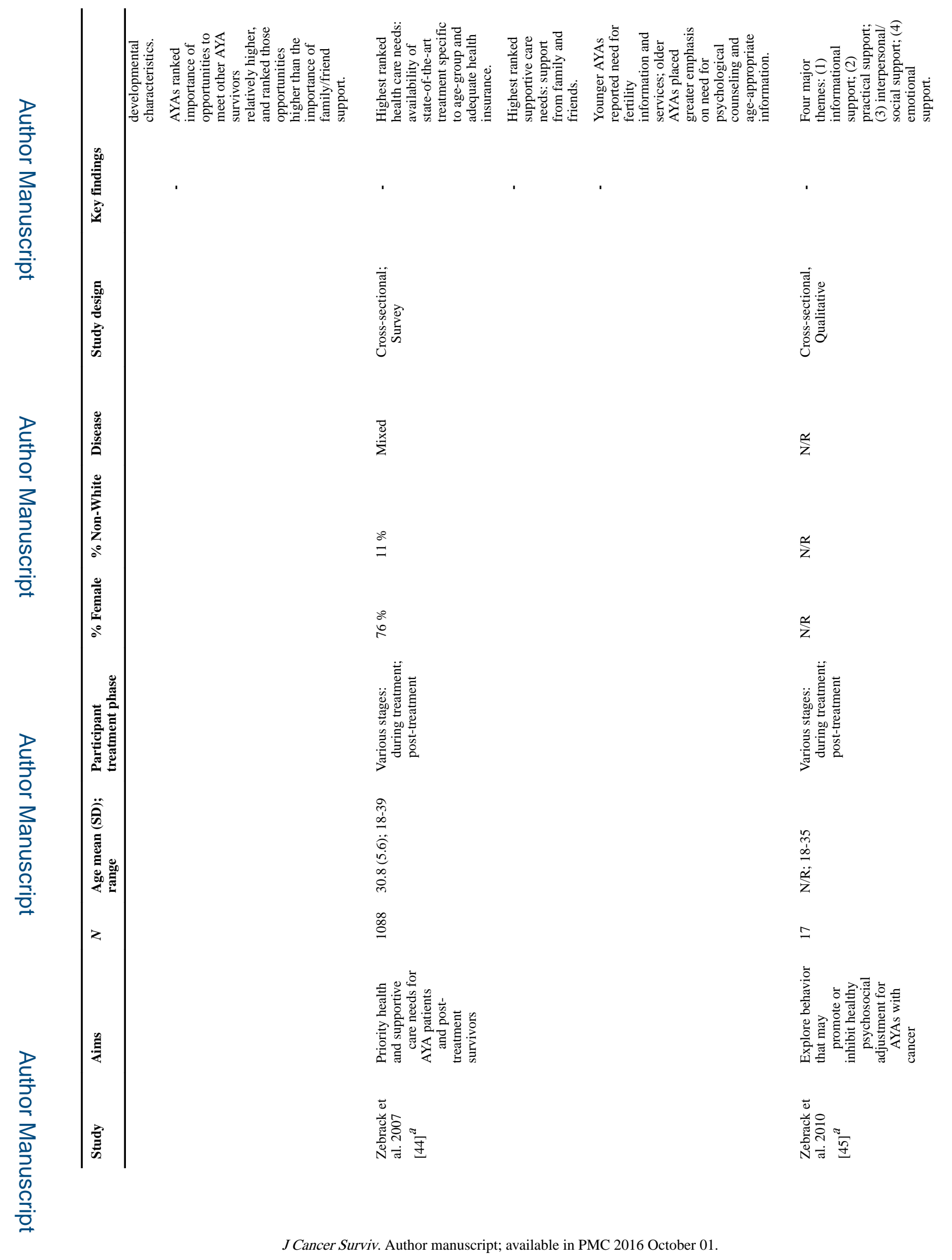


Barnett et al.

Page 37

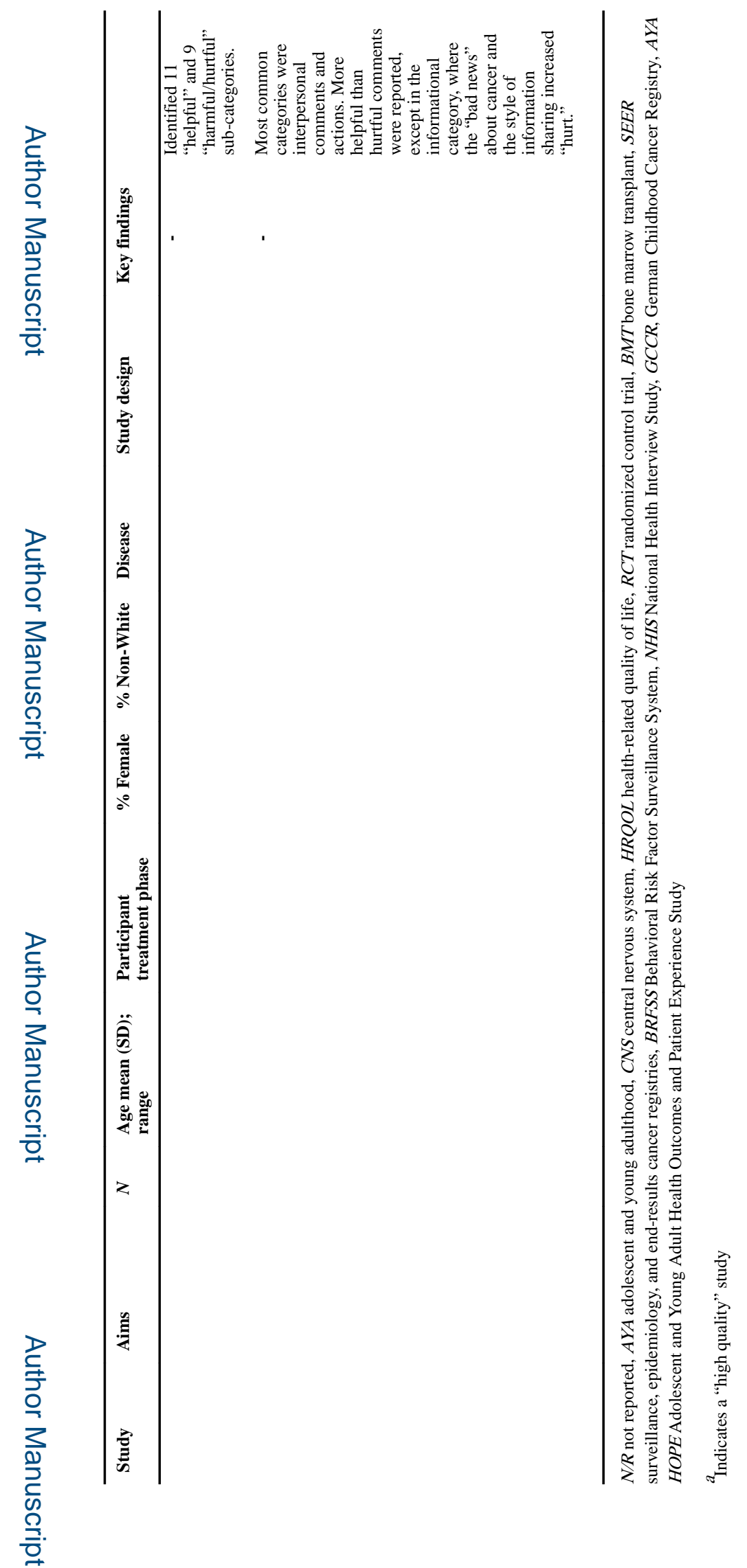

J Cancer Surviv. Author manuscript; available in PMC 2016 October 01. 